\title{
THE FIXED POINT PROPERTY AND THE OPIAL CONDITION ON TREE-LIKE BANACH SPACES
}

\author{
COSTAS POULIOS
}

\begin{abstract}
We introduce some new tree-like Banach spaces, belonging to the class of separable Banach spaces not containing $\ell_{1}$ with non-separable dual, each one of which satisfies the following: (1) the space has the fixed point property and (2) the space does not satisfy the Opial condition. In addition, one of these spaces contains subspaces isomorphic to $c_{0}$, whose Banach-Mazur distance from $c_{0}$ becomes arbitrarily large.
\end{abstract}

1. Introduction. Suppose that $K$ is a weakly compact and convex subset of a Banach space $X$. A mapping $T: K \rightarrow K$ is said to be non-expansive if $\|T x-T y\| \leq\|x-y\|$ for any $x, y \in K$. In the case where every non-expansive self mapping $T: K \rightarrow K$ has a fixed point, we say that $K$ has the fixed point property. The Banach space $X$ is said to have the fixed point property if every weakly compact, convex subset $K$ of $X$ has the fixed point property.

Alspach's example (see [1]) showed that the space $L_{1}[0,1]$ fails the fixed point property. On the other hand, many positive results are known. The earlier of these results are due to Browder [3], who proved that any uniformly convex Banach space enjoys the fixed point property and to Kirk [10], who showed that normal structure also implies the fixed point property.

In the proofs of positive results, the notion of minimal invariant sets is highly used. Suppose that $K$ is a weakly compact, convex set and $T: K \rightarrow K$ is a non-expansive mapping. A nonempty, weakly compact, convex subset $C$ of $K$ such that $T(C) \subseteq C$ is called minimal for $T$ if there is no strictly smaller weakly compact, convex subset of

2010 AMS Mathematics subject classification. Primary 47H10, 47H09, 46B03, $46 \mathrm{~B} 99$.

Keywords and phrases. Fixed point property, Opial condition, normal structure, dyadic tree, Banach spaces not containing $\ell_{1}$ with nonseparable dual.

Received by the editors on February 20, 2012, and in revised form on July 26, 2013.

DOI:10.1216/RMJ-2015-45-4-1245

Copyright (C)2015 Rocky Mountain Mathematics Consortium 
$C$ which is invariant under $T$. A straightforward application of Zorn's lemma implies that $K$ always contains minimal invariant subsets. So, a standard approach in proving fixed point theorems is to first assume that $K$ itself is minimal for $T$ and then use the geometrical properties of the space to show that $K$ must be a singleton. Therefore, $T$ has a fixed point.

Although a non-expansive map $T: K \rightarrow K$ does not have to have fixed points, it is well known that $T$ always has an approximate fixed point sequence. This means that there is a sequence $\left(x_{n}\right)$ in $K$ such that $\lim _{n \rightarrow \infty}\left\|x_{n}-T x_{n}\right\|=0$. For such sequences, the following result holds (see [8]).

Theorem 1.1. Let $K$ be a weakly compact, convex set in a Banach space, $T: K \rightarrow K$ a non-expansive map, such that $K$ is $T$-minimal, and let $\left(x_{n}\right)$ be any approximate fixed point sequence. Then, for all $x \in K$,

$$
\lim _{n \rightarrow \infty}\left\|x-x_{n}\right\|=\operatorname{diam}(K) .
$$

We pass now to another property of Banach spaces, which was introduced by Opial [15] and is closely related to the fixed point property. A Banach space $X$ is said to satisfy the Opial condition if, whenever a sequence $\left(x_{n}\right)$ in $X$ converges weakly to $x_{0} \in X$, then $\liminf \left\|x_{n}-x_{0}\right\|<\liminf \left\|x_{n}-x\right\|$ for all $x \in X, x \neq x_{0}$. It is known that the Opial condition implies that the space $X$ has normal structure (see [5]) and therefore it has the fixed point property (a simple proof that the Opial condition implies the fixed point property can also be deduced directly by the definitions). On the other hand, the inverse implication is by no means true, as the next proposition shows.

Proposition 1.2. Let $X$ be a Banach space with the Opial condition. Assume also that there is a sequence $\left(x_{n}\right)$ in $X$ such that $\left(x_{n}\right)$ converges weakly to 0 but it does not converge in norm. Consider the direct sum $Y=(X \oplus X)_{0}$ endowed with the maximum norm, that is, $\|(x, z)\|=$ $\max \{\|x\|,\|z\|\}$ for any $x, z \in X$. Then the space $Y$ does not satisfy the Opial condition; however, $Y$ has the fixed point property.

Further examples are also given by the spaces $L_{p}, 1<p<\infty p \neq 2$ (see [15]) and the space $c_{0}$. It is straightforward to verify that $c_{0}$ does 
not satisfy the Opial condition. On the other hand, the fact that $c_{0}$ has the fixed point property is due to Maurey [14] (see also [4]) and is based on the following fundamental result.

Theorem 1.3. Let $K$ be a weakly compact convex subset of a Banach space which is minimal for the non-expansive map $T: K \rightarrow K$. Let $\left(x_{n}\right)$ and $\left(y_{n}\right)$ be approximate fixed point sequences for $T$ such that $\lim _{n \rightarrow \infty}\left\|x_{n}-y_{n}\right\|$ exists. Then there is an approximate fixed point sequence $\left(z_{n}\right)$ in $K$ such that

$$
\lim _{n \rightarrow \infty}\left\|x_{n}-z_{n}\right\|=\lim _{n \rightarrow \infty}\left\|y_{n}-z_{n}\right\|=\frac{1}{2} \lim _{n \rightarrow \infty}\left\|x_{n}-y_{n}\right\| .
$$

In the present work, we consider the important class of separable Banach spaces not containing $\ell_{1}$ with nonseparable dual, and we study the Opial condition, the fixed point property and the relation between them in connection with the members of the aforementioned class. This class was established by two fundamental examples. The first one is the James tree space $(J T)$ invented by James [7], and the second one is the James Function space $(J F)$ due to Lindenstrauss and Stegall [13]. Among the many interesting properties which hold for the space $J T$, it has been proved that $J T$ satisfies the Opial condition $[\mathbf{9}, \mathbf{1 2}]$ (in fact, $J T$ satisfies the uniform Opial condition).

Consider now the direct sum $X=J T \oplus J T$ endowed with the maximum norm. It is known that $X$ is isomorphic to $J T$ and, therefore, $X$ belongs to the class that we have considered. Furthermore, according to Proposition 1.2, the space $X$ has the fixed point property although it does not satisfy the Opial condition. Therefore, the two properties are distinguished trivially by the space $(J T \oplus J T)_{0}$. The purpose of the present paper is to introduce some new nontrivial tree-like Banach spaces which also distinguish the properties under consideration.

We close this introductory section by recalling some notation and definitions concerning the standard dyadic tree $\mathcal{D}$, that is, the set $\mathcal{D}=\cup_{n=0}^{\infty}\{0,1\}^{n}$ of all finite sequences $s$ in $\{0,1\}$ including the empty sequence denoted by $\emptyset$. Elements $s \in \mathcal{D}$ are called nodes. If $s$ is a node and $s \in\{0,1\}^{n}$, we say $s$ is on the $n$th level of $\mathcal{D}$. We denote the level of a node $s$ by lev $(s)$. The initial segment partial ordering on $\mathcal{D}$ is denoted by $\leq$, and we write $s<s^{\prime}$ if $s \leq s^{\prime}$ and $s \neq s^{\prime}$. If 
$s, s^{\prime}$ are nodes such that neither $s \leq s^{\prime}$ nor $s^{\prime} \leq s$, then $s, s^{\prime}$ are called non-comparable and we write $s \perp s^{\prime}$.

A finite, linearly ordered subset $\mathcal{I}$ of $\mathcal{D}$ is called a segment if for every $s<t<s^{\prime}, t$ belongs to $\mathcal{I}$ provided that $s, s^{\prime}$ belong to $\mathcal{I}$. An infinite, linearly ordered subset $B$ of $\mathcal{D}$ satisfying the above property is called a branch. If lev $(\min B)=n$, we say $B$ is an $n$th branch.

A segment $\mathcal{I}$ is called initial if the empty sequence $\emptyset$ belongs to $\mathcal{I}$. For any node $s$, we set $\mathcal{I}(s)=\{t \in \mathcal{D} \mid t \leq s\}$. Clearly, $\mathcal{I}(s)$ is an initial segment. If $s, s^{\prime} \in \mathcal{D}$, the $\leq$-infimum of $\left\{s, s^{\prime}\right\}$ is defined by $s \wedge s^{\prime}=\max \left\{\mathcal{I}(s) \cap \mathcal{I}\left(s^{\prime}\right)\right\}$.

The lexicographical ordering on $\mathcal{D}$ denoted by $\leq_{\text {lex }}$ is defined as follows. For nodes $s, s^{\prime}$ we have $s \leq_{\operatorname{lex}} s^{\prime}$ if either $s \leq s^{\prime}$ or $s \perp s^{\prime}$, $t \frown 0 \leq s$ and $t \frown 1 \leq s^{\prime}$ where $t=s \wedge s^{\prime}$. We also write $s<\operatorname{lex} s^{\prime}$ whenever $s \leq_{\operatorname{lex}} s^{\prime}$ and $s \neq s^{\prime}$.

2. The case of the space $T F$. Before proceeding to the main part of this paper, we devote this section to the examination of the space $T F$ (see [2]). This space has been used to describe the structure of the subspaces of $J F$ which have nonseparable dual. The space $T F$ belongs to the class under consideration, that is it is separable, with nonseparable dual, and it does not contain $\ell_{1}$. It is also known that $T F$ contains isomorphs of $c_{0}$ and $\ell_{p}$ for any $p$ with $2 \leq p<\infty$. The presence of $c_{0}$ in the space $T F$ leads us to think that $T F$ may not have Opial's property. However, rather unexpectedly, $T F$ does satisfy the Opial condition. Therefore, $T F$ contains isomorphic copies of $c_{0}$ which are quite strange, at least from our point of view. In the following, we define the space $T F$ and we prove that it satisfies the Opial condition.

We start with the next definition. Suppose that $\mathcal{I}, \mathcal{I}_{1}, \mathcal{I}_{2}$ are (nonempty) segments of the dyadic tree $\mathcal{D}$. We set $a=\min \mathcal{I}, b=\max \mathcal{I}$, $a_{1}=\min \mathcal{I}_{1}$ and $a_{2}=\min \mathcal{I}_{2}$. We say that $\mathcal{I}$ separates the segments $\mathcal{I}_{1}$ and $\mathcal{I}_{2}$ if the following hold:

(1) $a \leq a_{1} \wedge a_{2}$,

(2) $b \perp a_{1}, b \perp a_{2}$ and either $a_{1}<_{\operatorname{lex}} b<_{\operatorname{lex}} a_{2}$ or $a_{2}<_{\operatorname{lex}} b<_{\operatorname{lex}} a_{1}$.

A family $\mathcal{S}$ of segments of $\mathcal{D}$ is called an (ns)-family if, for every $\mathcal{I}, \mathcal{I}_{1}$ and $\mathcal{I}_{2}$ in $\mathcal{S}$, we have that $\mathcal{I}$ does not separate $\mathcal{I}_{1}$ and $\mathcal{I}_{2}$. 
Consider now the vector space $c_{00}(\mathcal{D})$ of finitely supported functions $x: \mathcal{D} \rightarrow \mathbb{R}$. If $\mathcal{I}$ is any segment of $\mathcal{D}$, we set $\mathcal{I}^{*}(x)=\sum_{s \in \mathcal{I}} x(s)$. Then, for any $x \in c_{00}(\mathcal{D})$, we define the norm

$$
\|x\|_{T F}=\sup \left(\sum_{i=1}^{n}\left|\mathcal{I}_{i}^{*}(x)\right|^{2}\right)^{1 / 2}
$$

where the supremum is taken over all finite (ns)-families $\left\{\mathcal{I}_{i}\right\}_{i=1}^{n}$ of pairwise disjoint segments. The space $T F$ is the completion of $c_{00}(\mathcal{D})$ with respect to the norm defined above.

For any node $s \in \mathcal{D}$, the unit vector $e_{s}$ is defined by $e_{s}(t)=1$ if $t=s$ and $e_{s}(t)=0$ otherwise. We also consider the standard enumeration $\left\{s_{n}\right\}$ of the tree $\mathcal{D}$, that is, $n<m$ if either $\operatorname{lev}\left(s_{n}\right)<\operatorname{lev}\left(s_{m}\right)$ or $\operatorname{lev}\left(s_{n}\right)=\operatorname{lev}\left(s_{m}\right)$ and $s_{n}<_{\text {lev }} s_{m}$. Then the sequence $\left(e_{s_{n}}\right)_{n \in \mathbb{N}}$ is a Shauder basis for the space $T F$. Therefore, for every vector $x \in T F$, there is a unique sequence $\left(\lambda_{n}\right)_{n \in \mathbb{N}}$ of real numbers such that $x=\sum_{n=1}^{\infty} \lambda_{n} e_{s_{n}}$. For any level $m$, we define the projection $P_{m}: T F \rightarrow T F$ by

$$
P_{m}(x)=P_{m}\left(\sum_{n=1}^{\infty} \lambda_{n} e_{s_{n}}\right)=\sum_{\operatorname{lev}\left(s_{n}\right) \geq m} \lambda_{n} e_{s_{n}} .
$$

It is easy to verify that $P_{m}$ is a bounded projection of norm one. For more details concerning the space $T F$ we refer to [2]. We restrict ourselves to the following result.

Theorem 2.1. The space TF satisfies the Opial condition.

Proof. Let us suppose that $T F$ does not satisfy the Opial condition. Then there exist a sequence $\left(x_{n}\right)$ in $T F$ and a vector $x \in T F, x \neq 0$ such that $x_{n} \stackrel{w}{\rightarrow} 0, \liminf \left\|x_{n}\right\|_{T F}=1$ and $\liminf \left\|x_{n}-x\right\|_{T F} \leq 1$.

Let $s_{0}$ be a node such that $\left|x\left(s_{0}\right)\right|=\max \{|x(s)|: s \in \mathcal{D}\}$. Since $x \neq 0$, we have that $\left|x\left(s_{0}\right)\right|>0$. Now fix a positive integer $N \in \mathbb{N}$, and let $\epsilon=1 / N$. Then there exists a finitely supported vector $y_{N} \in T F$ so that $\left\|y_{N}-x\right\|_{T F}<\epsilon$. We also consider a level $m$ with $m>\operatorname{lev}\left(s_{0}\right)$ and $P_{m}\left(y_{N}\right)=0$.

The assumption that $\left(x_{n}\right)$ is weakly null implies that, for any sufficiently large $n \in \mathbb{N}$, we have $\left\|x_{n}-P_{m} x_{n}\right\|_{T F}<\epsilon$. For any such 
$n$, we consider an (ns)-family $\left\{\mathcal{I}_{i, n}\right\}_{i=1}^{r_{n}}$ consisting of pairwise disjoint segments such that

$$
\left(\sum_{i=1}^{r_{n}}\left|\mathcal{I}_{i, n}^{*}\left(x_{n}\right)\right|^{2}\right)^{1 / 2} \geq\left\|x_{n}\right\|_{T F}-\epsilon .
$$

Without loss of generality, we may assume that lev $\left(\min \mathcal{I}_{i, n}\right) \geq m$ for every $i=1,2, \ldots, r_{n}$ (otherwise, we replace $\mathcal{I}_{i, n}$ by $\mathcal{J}_{i, n}=\mathcal{I}_{i, n} \cap\{s \in$ $\mathcal{D} \mid \operatorname{lev}(s) \geq m\})$. We also set $\mathcal{I}=\left\{s_{0}\right\}$.

We now claim that the collection $\mathcal{S}=\{\mathcal{I}\} \cup\left\{\mathcal{I}_{i, n} \mid i=1,2, \ldots, r_{n}\right\}$ forms an (ns)-family of pairwise disjoint segments. Indeed, by the definition given at the beginning of Section 2, we conclude that, whenever a segment $\mathcal{I}$ separates two other segments, then $\mathcal{I}$ must contain at least two nodes. Hence, the segment $\mathcal{I}=\left\{s_{0}\right\}$ cannot separate anything and $\mathcal{S}$ is an (ns)-family. By this observation, we get the following:

$$
\begin{aligned}
\left\|x_{n}-y_{N}\right\|_{T F}^{2} & \geq \sum_{i=1}^{r_{n}}\left|\mathcal{I}_{i, n}^{*}\left(x_{n}-y_{N}\right)\right|^{2}+\left|\mathcal{I}^{*}\left(x_{n}-y_{N}\right)\right|^{2} \\
& =\sum_{i=1}^{r_{n}}\left|\mathcal{I}_{i, n}^{*}\left(x_{n}\right)\right|^{2}+\left|y_{N}\left(s_{0}\right)-x_{n}\left(s_{0}\right)\right|^{2} \\
& \geq\left(\left\|x_{n}\right\|_{T F}-\epsilon\right)^{2}+\left|y_{N}\left(s_{0}\right)-x_{n}\left(s_{0}\right)\right|^{2}
\end{aligned}
$$

On the other hand,

$$
\begin{aligned}
\left\|x_{n}-y_{N}\right\|_{T F} & \leq\left\|x_{n}-x\right\|_{T F}+\left\|x-y_{N}\right\|_{T F} \\
& \leq\left\|x_{n}-x\right\|_{T F}+\epsilon .
\end{aligned}
$$

Combining the two inequalities, we obtain:

$$
\left(\left\|x_{n}\right\|_{T F}-\epsilon\right)^{2}+\left|y_{N}\left(s_{0}\right)-x_{n}\left(s_{0}\right)\right|^{2} \leq\left(\left\|x_{n}-x\right\|_{T F}+\epsilon\right)^{2} .
$$

Since $\lim \inf \left\|x_{n}\right\|_{T F}=1, x_{n} \stackrel{w}{\rightarrow} 0$ and $\liminf \left\|x_{n}-x\right\|_{T F} \leq 1$, the above inequality implies that

$$
\left|y_{N}\left(s_{0}\right)\right| \leq 2 \sqrt{\epsilon}
$$

Finally, we have

$$
\begin{aligned}
\left|x\left(s_{0}\right)\right| & \leq\left|x\left(s_{0}\right)-y_{N}\left(s_{0}\right)\right|+\left|y_{N}\left(s_{0}\right)\right| \\
& \leq \epsilon+2 \sqrt{\epsilon} .
\end{aligned}
$$


Therefore, for any $N \in \mathbb{N}$,

$$
\left|x\left(s_{0}\right)\right| \leq \frac{1}{N}+\frac{2}{\sqrt{N}},
$$

and, letting $N$ tend to infinity, we have $x\left(s_{0}\right)=0$, which is a contradiction.

3. The definition of the space $X$. In this section, we introduce a new tree-like Banach space, and we show that it belongs to the class of separable Banach spaces with nonseparable dual which do not contain $\ell_{1}$. In Sections 4 and 5 we study the fixed point property, the Opial condition and the embedding of $c_{0}$ in the space $X$. We start with the following definition.

Definition 3.1. Let $\mathcal{S}$ be a finite family of pairwise disjoint segments of the dyadic tree. The family $\mathcal{S}$ is called admissible if for any segment $\mathcal{I} \in \mathcal{S}$ there exists at most one segment $\mathcal{I}^{\prime} \in \mathcal{S}$ such that $\min \mathcal{I}<\min \mathcal{I}^{\prime}$.

Admissible families (in the previous sense) have a much simpler geometry on the dyadic tree than (ns)-families, which were defined in Section 2. Indeed, loosely speaking, suppose that we want to build an admissible family on $\mathcal{D}$, and let us start with any segment $\mathcal{I}_{1}$. Then we are allowed to choose at most one segment $\mathcal{I}_{2}$ contained in the subtree $\mathcal{D}_{s_{1}}=\left\{s \mid s \geq s_{1}\right\}$ where $s_{1}=\min \mathcal{I}_{1}$. Assume that the segment $\mathcal{I}_{2}$ has been chosen and $\min \mathcal{I}_{1}<\min \mathcal{I}_{2}$. Then we are obliged to choose $\mathcal{I}_{3}$ such that $\min \mathcal{I}_{3} \perp \min \mathcal{I}_{1}$. Again, we are able to consider at most one segment $\mathcal{I}_{4}$ with $\min \mathcal{I}_{4}>\min \mathcal{I}_{3}$, and so on. Therefore, it is easy to draw the arbitrary admissible family.

Some remarks concerning admissible families follow. First, assume that $\mathcal{S}$ is an admissible family of pairwise disjoint segments and, for each $\mathcal{I} \in \mathcal{S}$, consider a segment $\mathcal{I}^{\prime}$ with $\mathcal{I}^{\prime} \subseteq \mathcal{I}$. Let $\mathcal{S}^{\prime}=\left\{\mathcal{I}^{\prime} \mid \mathcal{I} \in \mathcal{S}\right\}$. By the above definition (and the picture obtained by the previous discussion), it is easy to see that $\mathcal{S}^{\prime}$ is also an admissible family. Secondly, suppose that $\mathcal{S}$ and $\mathcal{S}^{\prime}$ are admissible families. Then the family $\mathcal{S} \cup \mathcal{S}^{\prime}$ need not be admissible, even if it consists of pairwise disjoint segments. 
Consider now the vector space $c_{00}(\mathcal{D})$. For any $x \in c_{00}(\mathcal{D})$, we define the norm

$$
\|x\|=\sup \left[\sum_{i=1}^{r}\left|\mathcal{I}_{i}^{*}(x)\right|^{2}\right]^{1 / 2}
$$

where the supremum is taken over all finite admissible families $\left\{\mathcal{I}_{i}\right\}_{i=1}^{r}$ of pairwise disjoint segments. The Banach space $X$ is the completion of $c_{00}(\mathcal{D})$ with respect to the norm we have just defined.

For any node $s \in \mathcal{D}$, the unit vector $e_{s} \in X$ is defined in the usual way. Then, it is easily verified that the sequence $\left(e_{s}\right)_{s \in \mathcal{D}}$, where the tree $\mathcal{D}$ is given its standard enumeration, is a normalized, monotone Shauder basis for the space $X$. Therefore, $X$ is separable. Furthermore, as in the case of the space $J T$, it is proved that the dual $X^{*}$ of $X$ is nonseparable.

In order to proceed to the study of the space $X$, we need first to establish some notation. Let $\left(e_{s}^{*}\right)_{s \in \mathcal{D}}$ denote the sequence of biorthogonal functionals associated with the basis $\left(e_{s}\right)_{s \in \mathcal{D}}$. We set $F=\overline{\operatorname{span}}\left\{e_{s}^{*} \mid\right.$ $s \in \mathcal{D}\}$ the subspace of $X^{*}$ generated by the sequence $\left(e_{s}^{*}\right)_{s \in \mathcal{D}}$. Also let $\Gamma$ denote the set of all maximal branches of the dyadic tree $\mathcal{D}$. Then $\Gamma$ has the cardinality of the continuum. For any branch $B$, we define the functional $B^{*}: X \rightarrow \mathbb{R}$ by

$$
B^{*}(x)=B^{*}\left(\sum_{s \in \mathcal{D}} \lambda_{s} e_{s}\right)=\sum_{s \in B} \lambda_{s} .
$$

Then it is easily shown that $B^{*}$ is a bounded linear functional of norm one. Finally, the function $P_{m}: X \rightarrow X$ with $P_{m}\left(\sum_{s \in \mathcal{D}} \lambda_{s} e_{s}\right)=$ $\sum_{\text {lev }(s) \geq m} \lambda_{s} e_{s}$ defines for any level $m$ a bounded linear projection on $X$ of norm one.

We can now quote the first result for the space $X$.

\section{Theorem 3.2.}

(i) The quotient space $X^{*} / F$ is isomorphic to $\ell_{2}(\Gamma)$.

(ii) The second dual $X^{* *}$ of $X$ is isomorphic to $F^{*} \oplus \ell_{2}(\Gamma)$.

Proof. The first part of the theorem is proved as the corresponding statement for the space $J T$ (see [13]). For the second part, we cannot imitate the arguments of $J T$, since the basis $\left(e_{s}\right)_{s \in \mathcal{D}}$ of the space $X$ 
is not boundedly complete. However, in this case, we can appeal to the arguments used in the so-called Hagler tree space (HT) (see [6]). This is a tree-like Banach space not containing $\ell_{1}$ with non separable dual and its basis is not boundedly complete. The second part of our theorem is proved in a similar way as [6, Lemma 9].

The second part of the above theorem implies that the second dual $X^{* *}$ has the cardinality of the continuum. Therefore, $X$ does not contain a subspace isomorphic to $\ell_{1}$.

One further consequence of Theorem 3.2 is that the dual $X^{*}$ is determined in some sense by the biorthogonal functionals $\left(e_{s}^{*}\right)_{s \in \mathcal{D}}$ and the functionals $\left(B^{*}\right)_{B \in \Gamma}$. More precisely, by the proof of Theorem 3.2, the next corollary follows which will be used in the sequel.

Corollary 3.3. Suppose that $\left(x_{n}\right)$ is a bounded sequence of elements of $X$. Then $\left(x_{n}\right)$ converges weakly to zero if and only if $e_{s}^{*}\left(x_{n}\right) \rightarrow 0$ for any $s \in \mathcal{D}$ and $B^{*}\left(x_{n}\right) \rightarrow 0$ for any branch $B \in \Gamma$.

We close this section with some observations concerning the subspaces of $X$. We first recall that a chain of $\mathcal{D}$ is an infinite linearly ordered subset of $\mathcal{D}$. An antichain is an infinite subset of $\mathcal{D}$ whose elements are pairwise non-comparable.

\section{Proposition 3.4.}

(i) For any chain $\left(s_{n}\right)_{n \in \mathbb{N}}$ of $\mathcal{D}$, the sequence $\left(e_{s_{n}}\right)_{n \in \mathbb{N}}$ is equivalent to the summing basis of $c_{0}$. Therefore, the space $\overline{\operatorname{span}}\left\{e_{s_{n}} \mid n \in \mathbb{N}\right\}$ is isomorphic to $c_{0}$.

(ii) For any antichain $\left(s_{n}\right)_{n \in \mathbb{N}}$ of $\mathcal{D}$, the sequence $\left(e_{s_{n}}\right)_{n \in \mathbb{N}}$ is equivalent to the usual basis of $\ell_{2}$ and the space $\overline{\operatorname{span}}\left\{e_{s_{n}} \mid n \in \mathbb{N}\right\}$ is isometrically isomorphic to $\ell_{2}$.

4. The fixed point property. This section is entirely devoted to the proof of the following theorem.

Theorem 4.1. The space $X$ has the fixed point property. 
Proof. Let $K$ be a weakly compact and convex subset of $X$, and let $T: K \rightarrow K$ be a non-expansive map. We assume that $K$ is minimal for $T$ and, using the geometry of the space $X$, we will prove that $\operatorname{diam}(K)=0$. Suppose on the contrary that $\operatorname{diam}(K)>0$. Without loss of generality, we may assume that $\operatorname{diam}(K)=1$.

Consider a sequence $\left(x_{n}\right)$ in $K$ such that $\lim _{n \rightarrow \infty}\left\|x_{n}-T x_{n}\right\|=0$. By passing to a subsequence and by translation, if necessary, we may assume that $\left(x_{n}\right)$ converges weakly to 0 . By Theorem 1.1, it follows that $\lim _{n \rightarrow \infty}\left\|x_{n}\right\|=\operatorname{diam}(K)=1$. We next define a subsequence $\left(x_{k_{n}}\right)=\left(y_{n}\right)$ of $\left(x_{n}\right)$ such that, for each $n$, the vectors $x_{n}$ and $y_{n}$ have essentially disjoint supports. The sequence $\left(y_{n}\right)$ is defined inductively as follows. Assume that we have found the elements $y_{1}=x_{k_{1}}, y_{2}=x_{k_{2}}, \ldots, y_{n-1}=x_{k_{n-1}}$. Consider now the vector $x_{n}$. We know that there is a level $N_{n}$ such that

$$
\left\|P_{N_{n}}\left(x_{n}\right)\right\|<\frac{1}{n} .
$$

Since $\left(x_{n}\right)$ converges weakly to 0 , we find $y_{n}=x_{k_{n}}$ with $k_{n}>k_{n-1}$ such that

$$
\left\|y_{n}-P_{N_{n}}\left(y_{n}\right)\right\|<\frac{1}{n} .
$$

After the sequence $\left(y_{n}\right)$ has been defined, we pass now to the following claim.

Claim. The limit $\lim _{n \rightarrow \infty}\left\|x_{n}-y_{n}\right\|$ is equal to 1 .

Indeed, we fix $n \in \mathbb{N}$, and we consider an admissible family $\left\{\mathcal{I}_{i}\right\}_{i=1}^{r}$ of pairwise disjoint segments such that

$$
\left\|x_{n}\right\|^{2}-1 / n<\sum_{i=1}^{r}\left|\mathcal{I}_{i}^{*}\left(x_{n}\right)\right|^{2} .
$$

We next prune these segments at the level $N_{n}$, and we set $\mathcal{J}_{i}=\mathcal{I}_{i} \cap\{s \mid$ $\left.\operatorname{lev}(s)<N_{n}\right\}$. Both families $\left\{\mathcal{J}_{i}\right\}_{i=1}^{r}$ and $\left\{\mathcal{I}_{i} \backslash \mathcal{J}_{i}\right\}_{i=1}^{r}$ are admissible. So, we obtain

$$
\left(\sum_{i=1}^{r}\left|\mathcal{I}_{i}^{*}\left(x_{n}\right)\right|^{2}\right)^{1 / 2} \leq\left(\sum_{i=1}^{r}\left|\mathcal{J}_{i}^{*}\left(x_{n}\right)\right|^{2}\right)^{1 / 2}+\left(\sum_{i=1}^{r}\left|\left(\mathcal{I}_{i} \backslash \mathcal{J}_{i}\right)^{*}\left(x_{n}\right)\right|^{2}\right)^{1 / 2}
$$




$$
\begin{aligned}
& \leq\left(\sum_{i=1}^{r}\left|\mathcal{J}_{i}^{*}\left(x_{n}\right)\right|^{2}\right)^{1 / 2}+\left\|P_{N_{n}}\left(x_{n}\right)\right\| \\
& \leq\left(\sum_{i=1}^{r}\left|\mathcal{J}_{i}^{*}\left(x_{n}\right)\right|^{2}\right)^{1 / 2}+\frac{1}{n} .
\end{aligned}
$$

Therefore,

$$
\begin{aligned}
\left\|x_{n}-y_{n}\right\| & \geq\left(\sum_{i=1}^{r}\left|\mathcal{J}_{i}^{*}\left(x_{n}\right)-\mathcal{J}_{i}^{*}\left(y_{n}\right)\right|^{2}\right)^{1 / 2} \\
& \geq\left(\sum_{i=1}^{r}\left|\mathcal{J}_{i}^{*}\left(x_{n}\right)\right|^{2}\right)^{1 / 2}-\left(\sum_{i=1}^{r}\left|\mathcal{J}_{i}^{*}\left(y_{n}\right)\right|^{2}\right)^{1 / 2} \\
& \geq\left(\sum_{i=1}^{r}\left|\mathcal{I}_{i}^{*}\left(x_{n}\right)\right|^{2}\right)^{1 / 2}-\frac{1}{n}-\left\|y_{n}-P_{N_{n}}\left(y_{n}\right)\right\| \\
& \geq\left(\left\|x_{n}\right\|^{2}-\frac{1}{n}\right)^{1 / 2}-\frac{2}{n},
\end{aligned}
$$

and the result follows.

We now fix a positive integer, $N$, which will be chosen properly at the end of the proof, and we set $\epsilon=2^{-N}$. By iterating applications of Theorem 1.3 , we find a sequence $\left(z_{n}\right)$ of elements of $K$, such that the following hold:

(i) $\left(z_{n}\right)$ is an approximate fixed point sequence for the mapping $T$. Therefore, by Theorem 1.1, $\lim _{n \rightarrow \infty}\left\|z_{n}\right\|=1$.

(ii) $\lim _{n \rightarrow \infty}\left\|z_{n}-x_{n}\right\|=1-\epsilon$ and $\lim _{n \rightarrow \infty}\left\|z_{n}-y_{n}\right\|=\epsilon$.

Without loss of generality, by omitting finitely many terms of the sequences, we may assume that, for each $n \in \mathbb{N}$, we have the following:

$$
\begin{aligned}
\left\|z_{n}\right\|^{2} & >1-\frac{\epsilon}{4} \\
1-\frac{5 \epsilon}{4} & <\left\|z_{n}-x_{n}\right\|<1-\frac{3 \epsilon}{4}, \\
\frac{3 \epsilon}{4} & <\left\|z_{n}-y_{n}\right\|<\frac{5 \epsilon}{4} \\
\frac{1}{n} & <\frac{\epsilon}{4} .
\end{aligned}
$$


By the definition of the norm in space $X$, it follows that there is an admissible family $\left\{\mathcal{I}_{i}\right\}_{i=1}^{r}$ of pairwise disjoint segments such that

$$
\sum_{i=1}^{r}\left|\mathcal{I}_{i}^{*}\left(z_{n}\right)\right|^{2}>1-\frac{\epsilon}{4} .
$$

We also have

$$
\sum_{i=1}^{r}\left|\mathcal{I}_{i}^{*}\left(z_{n}\right)\right|^{2} \leq\left\|z_{n}\right\|^{2} \leq 1 .
$$

Our next concern is to find out some more information about the place of the segments $\left\{\mathcal{I}_{i}\right\}_{i=1}^{r}$ on the dyadic tree. In particular, we consider the following cases.

Case 1. Assume that $\operatorname{lev}\left(\max \mathcal{I}_{i}\right)<N_{n}$ for any $i=1,2, \ldots, r$. Then we have:

$$
\begin{aligned}
\left(\sum_{i=1}^{r}\left|\mathcal{I}_{i}^{*}\left(z_{n}\right)\right|^{2}\right)^{1 / 2} & =\left(\sum_{i=1}^{r}\left|\mathcal{I}_{i}^{*}\left(z_{n}-y_{n}\right)+\mathcal{I}_{i}^{*}\left(y_{n}\right)\right|^{2}\right)^{1 / 2} \\
\leq & \left(\sum_{i=1}^{r}\left|\mathcal{I}_{i}^{*}\left(z_{n}-y_{n}\right)\right|^{2}\right)^{1 / 2} \\
& +\left(\sum_{i=1}^{r}\left|\mathcal{I}_{i}^{*}\left(y_{n}\right)\right|^{2}\right)^{1 / 2} \\
\leq & \left\|z_{n}-y_{n}\right\|+\left\|y_{n}-P_{N_{n}}\left(y_{n}\right)\right\| \\
\leq & \frac{5 \epsilon}{4}+\frac{\epsilon}{4} \\
= & \frac{6 \epsilon}{4}
\end{aligned}
$$

which is a contradiction.

Case 2. Assume that $\operatorname{lev}\left(\min \mathcal{I}_{i}\right) \geq N_{n}$ for each $i=1,2, \ldots, r$. As in Case 1, using the vector $x_{n}$ instead of $y_{n}$, we obtain:

$$
\begin{aligned}
\left(\sum_{i=1}^{r}\left|\mathcal{I}_{i}^{*}\left(z_{n}\right)\right|^{2}\right)^{1 / 2} & =\left(\sum_{i=1}^{r}\left|\mathcal{I}_{i}^{*}\left(z_{n}-x_{n}\right)+\mathcal{I}_{i}^{*}\left(x_{n}\right)\right|^{2}\right)^{1 / 2} \\
& \leq\left(\sum_{i=1}^{r}\left|\mathcal{I}_{i}^{*}\left(z_{n}-x_{n}\right)\right|^{2}\right)^{1 / 2}+\left(\sum_{i=1}^{r}\left|\mathcal{I}_{i}^{*}\left(x_{n}\right)\right|^{2}\right)^{1 / 2}
\end{aligned}
$$




$$
\begin{aligned}
& \leq\left\|z_{n}-x_{n}\right\|+\left\|P_{N_{n}}\left(x_{n}\right)\right\| \\
& \leq 1-\frac{3 \epsilon}{4}+\frac{\epsilon}{4} \\
& =1-\frac{\epsilon}{2},
\end{aligned}
$$

which is also a contradiction.

Case 3. Assume that there is a partition $\{1,2, \ldots, r\}=A_{1} \cup A_{2}$ such that lev $\left(\max \mathcal{I}_{i}\right)<N_{n}$ for each $i \in A_{1}$ and $\operatorname{lev}\left(\min \mathcal{I}_{i}\right) \geq N_{n}$ for each $i \in A_{2}$. Combining Cases 1 and 2, we obtain

$$
\begin{aligned}
\sum_{i=1}^{r}\left|\mathcal{I}_{i}^{*}\left(z_{n}\right)\right|^{2} & =\sum_{i \in A_{1}}\left|\mathcal{I}_{i}^{*}\left(z_{n}\right)\right|^{2}+\sum_{i \in A_{2}}\left|\mathcal{I}_{i}^{*}\left(z_{n}\right)\right|^{2} \\
& \leq\left(\frac{3 \epsilon}{2}\right)^{2}+\left(1-\frac{\epsilon}{2}\right)^{2} \\
& =1+\frac{5 \epsilon^{2}}{2}-\epsilon .
\end{aligned}
$$

If $\epsilon$ is small enough $(\epsilon<3 / 10)$, then we have

$$
1+\frac{5 \epsilon^{2}}{2}-\epsilon<1-\frac{\epsilon}{4}
$$

and we have reached a contradiction.

By the above discussion, we conclude that the admissible family $\left\{\mathcal{I}_{i}\right\}_{i=1}^{r}$ must contain at least one segment $\mathcal{I}_{i}$ which passes through the level $N_{n}$, in the sense that $\operatorname{lev}\left(\min \mathcal{I}_{i}\right)<N_{n} \leq \operatorname{lev}\left(\max \mathcal{I}_{i}\right)$. We now set:

$$
\begin{aligned}
& A_{1}=\left\{i \mid \operatorname{lev}\left(\max \mathcal{I}_{i}\right)<N_{n}\right\} \\
& A_{2}=\left\{i \mid \operatorname{lev}\left(\min \mathcal{I}_{i}\right) \geq N_{n}\right\} \\
& A_{3}=\left\{i \mid \operatorname{lev}\left(\min \mathcal{I}_{i}\right)<N_{n} \leq \operatorname{lev}\left(\max \mathcal{I}_{i}\right)\right\}
\end{aligned}
$$

Then, $A_{3}$ is nonempty. For each $i \in A_{3}$, we divide the segment $\mathcal{I}_{i}$ into two segments, as follows:

$$
\begin{aligned}
& E_{i}=\mathcal{I}_{i} \cap\left\{s \in \mathcal{D} \mid \operatorname{lev}(s)<N_{n}\right\} \\
& K_{i}=\mathcal{I}_{i} \cap\left\{s \in \mathcal{D} \mid \operatorname{lev}(s) \geq N_{n}\right\} .
\end{aligned}
$$

It is easy to observe that both $\left\{\mathcal{I}_{i}\right\}_{i \in A_{1}} \cup\left\{E_{i}\right\}_{i \in A_{3}}$ and $\left\{\mathcal{I}_{i}\right\}_{i \in A_{2}} \cup$ $\left\{K_{i}\right\}_{i \in A_{3}}$ are admissible families of pairwise disjoint segments. Now, 
for some $\delta>0$, which will be chosen properly, and using Cases 1 and 2, we obtain:

$$
\begin{aligned}
1-\frac{\epsilon}{4}< & \sum_{i=1}^{r}\left|\mathcal{I}_{i}^{*}\left(z_{n}\right)\right|^{2}=\sum_{i \in A_{1}}\left|\mathcal{I}_{i}^{*}\left(z_{n}\right)\right|^{2}+\sum_{i \in A_{2}}\left|\mathcal{I}_{i}^{*}\left(z_{n}\right)\right|^{2}+\sum_{i \in A_{3}}\left|\mathcal{I}_{i}^{*}\left(z_{n}\right)\right|^{2} \\
= & \sum_{i \in A_{1}}\left|\mathcal{I}_{i}^{*}\left(z_{n}\right)\right|^{2}+\sum_{i \in A_{2}}\left|\mathcal{I}_{i}^{*}\left(z_{n}\right)\right|^{2}+\sum_{i \in A_{3}}\left|E_{i}^{*}\left(z_{n}\right)+K_{i}^{*}\left(z_{n}\right)\right|^{2} \\
= & \sum_{i \in A_{1}}\left|\mathcal{I}_{i}^{*}\left(z_{n}\right)\right|^{2}+\sum_{i \in A_{2}}\left|\mathcal{I}_{i}^{*}\left(z_{n}\right)\right|^{2} \\
& +\left(1+\frac{1}{\delta}\right) \sum_{i \in A_{3}}\left|E_{i}^{*}\left(z_{n}\right)\right|^{2}+(1+\delta) \sum_{i \in A_{3}}\left|K_{i}^{*}\left(z_{n}\right)\right|^{2} \\
= & \left(\sum_{i \in A_{1}}\left|\mathcal{I}_{i}^{*}\left(z_{n}\right)\right|^{2}+\sum_{i \in A_{3}}\left|E_{i}^{*}\left(z_{n}\right)\right|^{2}\right) \\
& +\left(\sum_{i \in A_{2}}\left|\mathcal{I}_{i}^{*}\left(z_{n}\right)\right|^{2}+\sum_{i \in A_{3}}\left|K_{i}^{*}\left(z_{n}\right)\right|^{2}\right) \\
& +\frac{1}{\delta} \sum_{i \in A_{3}}\left|E_{i}^{*}\left(z_{n}\right)\right|^{2}+\delta \sum_{i \in A_{3}}\left|K_{i}^{*}\left(z_{n}\right)\right|^{2} \\
\leq & \left(\frac{3 \epsilon}{2}\right)^{2}+\left(1-\frac{\epsilon}{2}\right)^{2}+\frac{1}{\delta}\left(\frac{3 \epsilon}{2}\right)^{2}+\delta\left(1-\frac{\epsilon}{2}\right)^{2} .
\end{aligned}
$$

The function

$$
f(\delta)=\left(\frac{3 \epsilon}{2}\right)^{2}+\left(1-\frac{\epsilon}{2}\right)^{2}+\frac{1}{\delta}\left(\frac{3 \epsilon}{2}\right)^{2}+\delta\left(1-\frac{\epsilon}{2}\right)^{2}
$$

attains its minimum value for $\delta=(3 \epsilon / 2) /(1-\epsilon / 2)$. For this choice of $\delta$, we have $f(\delta)=(1+\epsilon)^{2}$. It follows that each one of the above inequalities brings an increase not bigger than

$$
(1+\epsilon)^{2}-\left(1-\frac{\epsilon}{4}\right)=\epsilon^{2}+2 \epsilon+\frac{\epsilon}{4} .
$$

In particular,

$$
\left(1-\frac{\epsilon}{2}\right)^{2}-\sum_{i \in A_{3}}\left|K_{i}^{*}\left(z_{n}\right)\right|^{2}<\epsilon^{2}+2 \epsilon+\frac{\epsilon}{4},
$$


which implies that

$$
\sum_{i \in A_{3}}\left|K_{i}^{*}\left(z_{n}\right)\right|^{2}>1-5 \epsilon .
$$

Consequently, we have proved that a large part of the norm of $z_{n}$ is given by the family $\left\{K_{i}\right\}_{i \in A_{3}}$. Since $z_{n}$ is close to $y_{n}$, it follows that a similar result holds for the vector $y_{n}$. More specifically, we have

$$
\begin{aligned}
\frac{5 \epsilon}{4} & >\left\|z_{n}-y_{n}\right\| \geq\left(\sum_{i \in A_{3}}\left|K_{i}^{*}\left(z_{n}-y_{n}\right)\right|^{2}\right)^{1 / 2} \\
& \geq\left(\sum_{i \in A_{3}}\left|K_{i}^{*}\left(z_{n}\right)\right|^{2}\right)^{1 / 2}-\left(\sum_{i \in A_{3}}\left|K_{i}^{*}\left(y_{n}\right)\right|^{2}\right)^{1 / 2} \\
& >(1-5 \epsilon)^{1 / 2}-\left(\sum_{i \in A_{3}}\left|K_{i}^{*}\left(y_{n}\right)\right|^{2}\right)^{1 / 2} \\
& >1-5 \epsilon-\left(\sum_{i \in A_{3}}\left|K_{i}^{*}\left(y_{n}\right)\right|^{2}\right)^{1 / 2} .
\end{aligned}
$$

Therefore,

$$
\left(\sum_{i \in A_{3}}\left|K_{i}^{*}\left(y_{n}\right)\right|^{2}\right)^{1 / 2} \geq 1-7 \epsilon
$$

At this point, let us summarize what we have proved so far. Firstly we have found approximate fixed point sequences $\left(x_{n}\right)$ and $\left(y_{n}\right)$ such that, for each $n$, the vectors $x_{n}$ and $y_{n}$ have essentially disjoint supports. Further, for each $n$, we have found pairwise disjoint segments which depend on $n$, and, for this reason, we now use the notation $\left\{K_{i, n}\right\}_{i=1}^{m_{n}}$, such that

(i) $\left(\sum_{i=1}^{m_{n}}\left|K_{i, n}^{*}\left(y_{n}\right)\right|^{2}\right)^{1 / 2} \geq 1-7 \epsilon$

(ii) for each $i$, the minimum node of $K_{i, n}$ lies on the level $N_{n}$.

Clearly, property (ii) is the most important. Finding just an admissible family whose segments start below the level $N_{n}$ and satisfy property (i) is quite easy since it is an immediate consequence of the definition of the norm. However, property (ii) gives much more information about the place of $\left\{K_{i, n}\right\}_{i=1}^{m_{n}}$ on the dyadic tree. We also notice that we will no longer make use of the sequence $\left(z_{n}\right)$. We used it in order to derive 
the previous result concerning the sequences $\left(x_{n}\right)$ and $\left(y_{n}\right)$, and we now just forget it.

We next choose a further subsequence $\left(y_{n}^{\prime}\right)=\left(x_{l_{n}}\right)$ of $\left(x_{n}\right)$, and we repeat the previous arguments to the $\left(y_{n}^{\prime}\right)$. First, the sequence $\left(y_{n}^{\prime}\right)$ is defined inductively as follows. Assume that we have chosen the vectors $y_{1}^{\prime}=x_{l_{1}}, \ldots, y_{n-1}^{\prime}=x_{l_{n-1}}$. Consider now the vector $x_{n}$ and the level $N_{n}$ for which we know that $\left\|P_{N_{n}}\left(x_{n}\right)\right\|<1 / n$. Since $\left(x_{n}\right)$ is weakly null, it follows that, for any segment $\mathcal{I}$, we have $\mathcal{I}^{*}\left(x_{n}\right) \rightarrow 0$. Therefore, there is an integer $l_{n}>l_{n-1}$ such that the term $y_{n}^{\prime}=x_{l_{n}}$ satisfies the following:

(a) $\left\|y_{n}^{\prime}-P_{N_{n}}\left(y_{n}^{\prime}\right)\right\|<1 / n$, that is, $x_{n}$ and $y_{n}^{\prime}$ have essentially disjoint supports, and

(b) $\left(\sum\left|\mathcal{I}^{*}\left(y_{n}^{\prime}\right)\right|^{2}\right)^{1 / 2}<\epsilon$, where the sum is taken over all the segments $\mathcal{I}$ such that $\mathcal{I} \subseteq K_{i, n}$ for some $i=1, \ldots, m_{n}$.

Note that, for a fixed $n$, there are only finitely many segments $\mathcal{I}$ such that $\mathcal{I} \subseteq \cup_{i=1}^{m_{n}} K_{i, n}$, and therefore the choice of $y_{n}^{\prime}$ is possible.

Now repeat the first part of the proof for the sequences $\left(x_{n}\right)$ and $\left(y_{n}^{\prime}\right)$. In this way, for each $n$, we find an admissible family of pairwise disjoint segments $\left\{\Lambda_{j, n}\right\}_{j=1}^{\mu_{n}}$ such that

(i) $\left(\sum_{j=1}^{\mu_{n}}\left|\Lambda_{j, n}^{*}\left(y_{n}^{\prime}\right)\right|^{2}\right)^{1 / 2} \geq 1-7 \epsilon$

(ii) for each $j=1, \ldots, \mu_{n}$, the minimum node of $\Lambda_{j, n}$ lies on the level $N_{n}$.

Consider now a segment $\Lambda_{j, n}$ for some $j=1, \ldots, \mu_{n}$. Then, we do not know whether the sets $\Lambda_{j, n}$ and $\cup_{i=1}^{m_{n}} K_{i, n}$ are disjoint or not. There may be at most one $i=i(j) \in\left\{1,2, \ldots, m_{n}\right\}$ such that $\Lambda_{j, n} \cap K_{i(j), n} \neq \emptyset$. In this case, by the whole construction, we have that the segments $\Lambda_{j, n}$ and $K_{i(j), n}$ share the same minimum node and the intersection $\Lambda_{j, n} \cap K_{i(j), n}$ is an initial part of $\Lambda_{j, n}$. However, the choice of the sequence $\left(y_{n}^{\prime}\right)$ and, in particular, property (b) indicates that $\left|\left(\Lambda_{j, n} \cap K_{i(j), n}\right)^{*}\left(y_{n}^{\prime}\right)\right|$ is very small. So, if, for each $j=1, \ldots, \mu_{n}$, we set $\Gamma_{j, n}=\Lambda_{j, n} \backslash\left(\Lambda_{j, n} \cap K_{i(j), n}\right)$, then we have

$$
\left(\sum_{j=1}^{\mu_{n}}\left|\Gamma_{j, n}^{*}\left(y_{n}^{\prime}\right)\right|^{2}\right)^{1 / 2}=\left(\sum_{j=1}^{\mu_{n}}\left|\Lambda_{j, n}^{*}\left(y_{n}^{\prime}\right)-\left(\Lambda_{j, n} \cap K_{i(j), n}\right)^{*}\left(y_{n}^{\prime}\right)\right|^{2}\right)^{1 / 2}
$$




$$
\begin{aligned}
\geq & \left(\sum_{j=1}^{\mu_{n}}\left|\Lambda_{j, n}^{*}\left(y_{n}^{\prime}\right)\right|^{2}\right)^{1 / 2} \\
& -\left(\sum_{j=1}^{\mu_{n}}\left|\left(\Lambda_{j, n} \cap K_{i(j), n}\right)^{*}\left(y_{n}^{\prime}\right)\right|^{2}\right)^{1 / 2} \\
\geq & 1-7 \epsilon-\epsilon \\
= & 1-8 \epsilon .
\end{aligned}
$$

At this point, we can set $\mathcal{S}=\left\{K_{i, n}\right\}_{i=1}^{m_{n}} \cup\left\{\Gamma_{j, n}\right\}_{j=1}^{\mu_{n}}$. Then we observe that $\mathcal{S}$ consists of pairwise disjoint segments. What is more, for each segment of the form $K_{i, n}$, there is at most one segment of $\mathcal{S}$ whose minimum node is a follower of $\min K_{i, n}$. And, for each segment $\Gamma_{j, n}$, there is no segment in $\mathcal{S}$ so that its minimum node is a follower of $\min \Gamma_{j, n}$. That is, the family $\mathcal{S}$ is admissible.

Since $\mathcal{S}$ is admissible, we use the choice of the sequence $\left(y_{n}^{\prime}\right)$ (property (b)) to obtain:

$$
\begin{aligned}
\left\|y_{n}-y_{n}^{\prime}\right\|^{2} \geq & \sum_{j=1}^{\mu_{n}}\left|\Gamma_{j, n}^{*}\left(y_{n}-y_{n}^{\prime}\right)\right|^{2}+\sum_{i=1}^{m_{n}}\left|K_{i, n}^{*}\left(y_{n}-y_{n}^{\prime}\right)\right|^{2} \\
\geq & \sum_{j=1}^{\mu_{n}}\left|\Gamma_{j, n}^{*}\left(y_{n}-y_{n}^{\prime}\right)\right|^{2}+\sum_{i=1}^{m_{n}}\left|K_{i, n}^{*}\left(y_{n}\right)-K_{i, n}^{*}\left(y_{n}^{\prime}\right)\right|^{2} \\
\geq & \sum_{j=1}^{\mu_{n}}\left|\Gamma_{j, n}^{*}\left(y_{n}-y_{n}^{\prime}\right)\right|^{2} \\
& +\left[\left(\sum_{i=1}^{m_{n}}\left|K_{i, n}^{*}\left(y_{n}\right)\right|^{2}\right)^{1 / 2}-\left(\sum_{i=1}^{m_{n}}\left|K_{i, n}^{*}\left(y_{n}^{\prime}\right)\right|^{2}\right)^{1 / 2}\right]^{2} \\
\geq & \sum_{j=1}^{\mu_{n}}\left|\Gamma_{j, n}^{*}\left(y_{n}-y_{n}^{\prime}\right)\right|^{2}+(1-7 \epsilon-\epsilon)^{2} \\
= & \sum_{j=1}^{\mu_{n}}\left|\Gamma_{j, n}^{*}\left(y_{n}-y_{n}^{\prime}\right)\right|^{2}+(1-8 \epsilon)^{2} .
\end{aligned}
$$


On the other hand, $\left\|y_{n}-y_{n}^{\prime}\right\| \leq \operatorname{diam}(K)=1$. Therefore,

$$
\sum_{j=1}^{\mu_{n}}\left|\Gamma_{j, n}^{*}\left(y_{n}-y_{n}^{\prime}\right)\right|^{2} \leq 1-(1-8 \epsilon)^{2} \leq 16 \epsilon .
$$

Roughly speaking, the above inequality says that, for each $j, \Gamma_{j, n}^{*}\left(y_{n}\right)$ is close to $\Gamma_{j, n}^{*}\left(y_{n}^{\prime}\right)$. Since

$$
\left(\sum_{j=1}^{\mu_{n}}\left|\Gamma_{j, n}^{*}\left(y_{n}^{\prime}\right)\right|^{2}\right)^{1 / 2}>1-8 \epsilon,
$$

it follows that the sum

$$
\left(\sum_{j=1}^{\mu_{n}}\left|\Gamma_{j, n}^{*}\left(y_{n}\right)\right|^{2}\right)^{1 / 2}
$$

is also close to 1 , and therefore the norm of $y_{n}$ must be close to 2 . More precisely, we have

$$
\begin{aligned}
\left\|y_{n}\right\|^{2} \geq & \sum_{i=1}^{m_{n}}\left|K_{i, n}^{*}\left(y_{n}\right)\right|^{2}+\sum_{j=1}^{\mu_{n}}\left|\Gamma_{j, n}^{*}\left(y_{n}\right)\right|^{2} \\
\geq & (1-7 \epsilon)^{2} \\
& +\sum_{j=1}^{\mu_{n}}\left|\Gamma_{j, n}^{*}\left(y_{n}^{\prime}\right)+\Gamma_{j, n}^{*}\left(y_{n}-y_{n}^{\prime}\right)\right|^{2} \\
\geq & (1-7 \epsilon)^{2} \\
& +\left[\left(\sum_{j=1}^{\mu_{n}}\left|\Gamma_{j, n}^{*}\left(y_{n}^{\prime}\right)\right|^{2}\right)^{1 / 2}-\left(\sum_{j=1}^{\mu_{n}}\left|\Gamma_{j, n}^{*}\left(y_{n}-y_{n}^{\prime}\right)\right|^{2}\right)^{1 / 2}\right]^{2} \\
\geq & (1-7 \epsilon)^{2}+[(1-8 \epsilon)-4 \sqrt{\epsilon}]^{2} \\
\geq & 2-22 \sqrt{\epsilon} .
\end{aligned}
$$

Therefore, $\lim \left\|y_{n}\right\|^{2} \geq 2-22 \sqrt{\epsilon}$. On the other hand, $\lim \left\|y_{n}\right\|=$ $\operatorname{diam}(K)=1$. Hence, $1 \geq 2-22 \sqrt{\epsilon}$. If $\epsilon$ has been chosen small enough, the previous inequality does not hold, and we have reached a contradiction. Consequently, the space $X$ has the fixed point property. 


\section{Opial condition, normal structure and the embedding of} $c_{0}$ in the space $X$. We already know that the space $X$ contains a plethora of isomorphic copies of $c_{0}$. Indeed, if $B$ is any branch of the dyadic tree, then by Proposition 3.4, the space $\overline{\operatorname{span}}\left\{e_{s} \mid s \in B\right\}$, which will be denoted by $E$, is isomorphic to $c_{0}$. Further, it is not hard to prove that $E$ does not satisfy the Opial condition and therefore $X$ does not either. However, much more can be said concerning the embedding of $c_{0}$ in the space $X$.

Towards this direction, we first prove that there are subspaces $Y$ of $X$ isomorphic to $c_{0}$ such that the Banach-Mazur distance $d\left(Y, c_{0}\right)$ becomes arbitrarily large. Recall that, if $Y$ and $Z$ are isomorphic Banach spaces, then the Banach-Mazur distance $d(Y, Z)$ is defined by

$$
d(Y, Z)=\inf \left\{\|T\| \cdot\left\|T^{-1}\right\|: T \text { is an isomophism from } Y \text { onto } Z\right\} .
$$

Theorem 5.1. For any positive real number $M$ there exists a subspace $Y$ of $X$ such that $Y$ is isomorphic to $c_{0}$ and $d\left(Y, c_{0}\right)>M$.

Proof. For our convenience, we consider four distinct maximal branches $\left(B_{i}\right)_{i=0}^{3}$ of the tree $\mathcal{D}$. The general case of $N$ distinct branches is almost identical. Let $m$ be a level such that the sets $A_{i}=B_{i} \cap\{s \mid \operatorname{lev}(s) \geq m\}, i=0,1,2,3$, are pairwise disjoint. We also consider the following enumerations of the sets $\left\{A_{i}\right\}_{i=0}^{3}$ :

$$
\begin{aligned}
& A_{0}=\left\{s_{0}<s_{4}<s_{8}<\cdots\right\}=\left\{s_{4 k}\right\}_{k=0}^{\infty} \\
& A_{1}=\left\{s_{1}<s_{5}<s_{9}<\cdots\right\}=\left\{s_{4 k+1}\right\}_{k=0}^{\infty},
\end{aligned}
$$

and so on. Finally, we set $Y=\overline{\operatorname{span}}\left\{e_{s} \mid s \in \cup_{i=0}^{3} A_{i}\right\}=\overline{\operatorname{span}}\left\{e_{s_{n}} \mid\right.$ $n \in \mathbb{N}\}$. It is clear that $Y$ is isomorphic to the direct sum $E \oplus E \oplus E \oplus E$, and therefore it is isomorphic to $c_{0}$.

We next try to estimate the Banach-Mazur distance $d=d\left(Y, c_{0}\right)$. For any $\epsilon>0$, there is an isomorphism $T: Y \rightarrow c_{0}$ onto $c_{0}$ such that, for every $y \in Y$, we have

$$
\|y\| \leq\|T y\|_{c_{0}} \leq(d+\epsilon)\|y\|
$$

We now define a sequence $\left(y_{n}\right)$ in $Y$. Instead of giving a formal 
definition, we write down the first few terms of $\left(y_{n}\right)$ which go as follows:

$$
\begin{aligned}
& y_{0}=e_{s_{0}}-e_{s_{4}}, \quad y_{1}=e_{s_{1}}-e_{s_{5}}, \\
& y_{2}=e_{s_{2}}-e_{s_{6}}, \quad y_{3}=e_{s_{3}}-e_{s_{7}}, \\
& y_{4}=e_{s_{8}}-e_{s_{12}}, \quad y_{5}=e_{s_{9}}-e_{s_{13}},
\end{aligned}
$$

and we continue in an obvious manner. It is easy to see that $\left\|y_{n}\right\|=\sqrt{2}$ for each $n \in \mathbb{N}$. Moreover, by Corollary 3.3, it follows that $\left(y_{n}\right)$ converges weakly to 0 .

Let $u_{n}=T y_{n} \in c_{0}$ for each $n \in \mathbb{N}$. Since $T$ is w-w continuous, we have that $\left(u_{n}\right)$ is weakly null. By the inequality (5.1), we also have

$$
\sqrt{2} \leq\left\|u_{n}\right\|_{c_{0}} \leq(d+\epsilon) \sqrt{2} \text { for any } n \in \mathbb{N} .
$$

Let us write $u_{0}=\left(u_{0}(i)\right)_{i \in \mathbb{N}}$. Then there is an $i_{1} \in \mathbb{N}$ such that $\left|u_{0}(i)\right|<\epsilon$ for each $i \geq i_{1}$. Since the subsequence $\left(u_{4 k+1}\right)_{k \in \mathbb{N}}$ is weakly null, we find $k \in \mathbb{N}$ such that $\left|u_{4 k+1}(i)\right|<\epsilon$ for any $i<i_{1}$. Repeating this argument, we find $i_{2}>i_{1}$ such that $\left|u_{4 k+1}(i)\right|<\epsilon$ for any $i \geq i_{2}$, and an integer $l$ such that $\left|u_{4 l+2}(i)\right|<\epsilon$ for any $i<i_{2}$. The integers $i_{3}$ and $m$ are also chosen so that $i_{3}>i_{2},\left|u_{4 l+2}(i)\right|<\epsilon$ for any $i \geq i_{3}$ and $\left|u_{4 m+3}(i)\right|<\epsilon$ for any $i<i_{3}$. The inequality (5.2) and the above construction imply that, for each $i \in \mathbb{N}$,

$$
\left|u_{0}(i)+u_{4 k+1}(i)+u_{4 l+2}(i)+u_{4 m+3}(i)\right| \leq(d+\epsilon) \sqrt{2}+3 \epsilon .
$$

Therefore,

$$
\left\|u_{0}+u_{4 k+1}+u_{4 l+2}+u_{4 m+3}\right\|_{c_{0}} \leq(d+\epsilon) \sqrt{2}+3 \epsilon .
$$

On the other hand,

$$
\begin{aligned}
& \left\|u_{0}+u_{4 k+1}+u_{4 l+2}+u_{4 m+3}\right\|_{c_{0}} \\
& \quad=\left\|T\left(y_{0}+y_{4 k+1}+y_{4 l+2}+y_{4 m+3}\right)\right\|_{c_{0}} \\
& \quad \geq\left\|y_{0}+y_{4 k+1}+y_{4 l+2}+y_{4 m+3}\right\| .
\end{aligned}
$$

Now consider the admissible family $\left\{\mathcal{I}_{j}\right\}_{j=1}^{8}$ where each $\mathcal{I}_{j}$ is a singleton consisting of one node, $s$, belonging to the support of some of the vectors $y_{0}, y_{4 k+1}, y_{4 l+2}, y_{4 m+3}$. Then it is easy to see that $\mid \mathcal{I}_{j}^{*}\left(y_{0}+\right.$ $\left.y_{4 k+1}+y_{4 l+2}+y_{4 m+3}\right) \mid=1$ and

$$
\left\|y_{0}+y_{4 k+1}+y_{4 l+2}+y_{4 m+3}\right\| \geq \sqrt{8} .
$$


Consequently,

$$
\sqrt{8} \leq(d+\epsilon) \sqrt{2}+3 \epsilon
$$

and, when $\epsilon$ tends to 0 , we get that $d \geq \sqrt{4}$.

If we start with $N$ distinct branches, then the inequality (5.3) becomes

$$
\sqrt{2 N} \leq(d+\epsilon) \sqrt{2}+(N-1) \epsilon,
$$

which implies that $\sqrt{N} \leq d$, and the desired result follows.

A long-standing question in metric fixed point theory is the following. Find a non-trivial class of Banach spaces invariant under isomorphisms such that each member of the class has the fixed point property. (The trivial example is given by the spaces isomorphic to $\ell_{1}$.) By the above theorem and the result of Section 4, we obtain the next corollary.

Corollary 5.2. For any real number $M>0$, there exists a Banach space $Y$ isomorphic to $c_{0}$ such that $d\left(Y, c_{0}\right)>M$, and $Y$ has the fixed point property.

We do not know whether the space $c_{0}$ solves the aforementioned problem. What we say is that we can go as far away from $c_{0}$ as we want (in the sense of Banach-Mazur distance), and we find spaces with the fixed point property.

We now continue the investigation of the embedding of $c_{0}$ in the space $X$. Our goal is to prove that there are subspaces of $X$ isomorphic to $c_{0}$ which are not generated by finitely many branches. (This argument will be useful to us in Section 6, where we introduce a second tree-like Banach space, and we show that it also contains isomorphic copies of $c_{0}$.) For this reason, we consider the sequence $\left(x_{n}\right)$ in $X$ where, for each $n \in \mathbb{N}, x_{n}: \mathcal{D} \rightarrow \mathbb{R}$ is defined as follows:

$$
x_{n}(s)=\left\{\begin{array}{cl}
\frac{1}{2^{n / 2}} & \text { if lev }(s)=n ; \\
0 & \text { otherwise. }
\end{array}\right.
$$

It is easy to see that $\left\|x_{n}\right\|=1$ for any $n \in \mathbb{N}$. For this sequence, we have the following. 
Theorem 5.3. The sequence $\left(x_{n}\right)$ is equivalent to the usual basis of $c_{0}$.

Proof. It suffices to prove that, for any $n \in \mathbb{N}$ and any $t_{1}, \ldots, t_{n} \in \mathbb{R}$, the following inequalities hold:

$$
\max _{1 \leq i \leq n}\left|t_{i}\right| \leq\left\|\sum_{i=1}^{n} t_{i} x_{i}\right\| \leq \sqrt{3}(\sqrt{2}+1) \max _{1 \leq i \leq n}\left|t_{i}\right| .
$$

The proof of the left-hand inequality is straightforward. For any fixed $k \in\{1,2, \ldots, n\}$, we set $\mathcal{I}_{j}=\left\{s_{j}\right\}, j=1,2, \ldots, 2^{k}$, where $\left\{s_{1}, s_{2}, \ldots, s_{2^{k}}\right\}$ is an enumeration of the nodes on the level $k$. Then, for each $j$,

$$
\mathcal{I}_{j}^{*}\left(\sum_{i=1}^{n} t_{i} x_{i}\right)=t_{k} \frac{1}{2^{k / 2}},
$$

and $\left\{\mathcal{I}_{j}\right\}_{j=1}^{2^{k}}$ is an admissible family of pairwise disjoint segments. Therefore,

$$
\left\|\sum_{i=1}^{n} t_{i} x_{i}\right\|^{2} \geq \sum_{j=1}^{2^{k}}\left|\mathcal{I}_{j}^{*}\left(\sum_{i=1}^{n} t_{i} x_{i}\right)\right|^{2}=\sum_{j=1}^{2^{k}}\left(\frac{t_{k}}{2^{k / 2}}\right)^{2}=t_{k}^{2} .
$$

Thus,

$$
\left\|\sum_{i=1}^{n} t_{i} x_{i}\right\| \geq \max _{1 \leq i \leq n}\left|t_{i}\right| .
$$

We proceed now to the right-hand inequality. The following claim is the main argument for our proof.

Claim 5.4. For any $n \in \mathbb{N}$, we have

$$
\left\|x_{0}+x_{1}+\cdots+x_{n}\right\| \leq \sqrt{3}(\sqrt{2}+1) .
$$

Proof of the claim. Fix $n \in \mathbb{N}$, and let $x=x_{0}+x_{1}+\cdots+x_{n}$. In order to prove the claim, we need to show that, for any admissible family $\mathcal{S}$ of pairwise disjoint segments, we have

$$
\sum_{\mathcal{I} \in \mathcal{S}}\left|\mathcal{I}^{*}(x)\right|^{2} \leq 3(\sqrt{2}+1)^{2} .
$$


We start with a special case. Suppose that the family $\mathcal{S}$ consists of two segments $\mathcal{I}_{1}$ and $\mathcal{I}_{2}$ such that $\min \mathcal{I}_{1}<\min \mathcal{I}_{2}$ and $\operatorname{lev}\left(\min \mathcal{I}_{1}\right)=m$. Then we have

$$
\begin{aligned}
\left|\mathcal{I}_{1}^{*}(x)\right|^{2}+\left|\mathcal{I}_{2}^{*}(x)\right|^{2} & \leq\left(\sum_{k=m}^{\infty} \frac{1}{(\sqrt{2})^{k}}\right)^{2}+\left(\sum_{k=m+1}^{\infty} \frac{1}{(\sqrt{2})^{k}}\right)^{2} \\
& =\frac{2(\sqrt{2}+1)^{2}}{2^{m}}+\frac{2(\sqrt{2}+1)^{2}}{2^{m+1}} \\
& =\frac{3(\sqrt{2}+1)^{2}}{2^{m}} .
\end{aligned}
$$

Now consider an arbitrary admissible family $\mathcal{S}$. We set $m_{1}=$ $\min \{\operatorname{lev}(\min \mathcal{I}) \mid \mathcal{I} \in \mathcal{S}\}$. We also assume that there are $k_{1}$ segments, say $\mathcal{I}_{1}, \ldots, \mathcal{I}_{k_{1}}$, belonging to the family $\mathcal{S}$ such that, for each $i$, the minimum node $s_{i}$ of $\mathcal{I}_{i}$ lies on the level $m_{1}$. Clearly, $k_{1} \leq 2^{m_{1}}$. Since $\mathcal{S}$ is admissible, for every $\mathcal{I}_{i}$ there is at most one segment $\mathcal{I}_{i}^{\prime} \in \mathcal{S}$ with $\min \mathcal{I}_{i}<\min \mathcal{I}_{i}^{\prime}$. Let $\mathcal{S}_{1}$ be the subfamily of $\mathcal{S}$ consisting of the segments $\left\{\mathcal{I}_{i}\right\}_{i=1}^{k_{1}}$ and the segments $\left\{\mathcal{I}_{i}^{\prime}\right\}_{i=1}^{k_{1}}$ (as many as there exist). Then by the special case studied above, we obtain

$$
\sum_{\mathcal{I} \in \mathcal{S}_{1}}\left|\mathcal{I}^{*}(x)\right|^{2} \leq \frac{k_{1}}{2^{m_{1}}} 3(\sqrt{2}+1)^{2} .
$$

We next set $m_{2}=\min \left\{\operatorname{lev}(\min \mathcal{I}) \mid \mathcal{I} \in \mathcal{S} \backslash \mathcal{S}_{1}\right\}$, and we assume that there are $k_{2}$ segments $\mathcal{I}$ such that $\mathcal{I} \in \mathcal{S} \backslash \mathcal{S}_{1}$ and $\operatorname{lev}(\min \mathcal{I})=m_{2}$. Observe that, for such a segment $\mathcal{I}$, its minimum node cannot be a follower of the $k_{1}$ nodes on the level $m_{1}$ which were found at the previous step, namely, the nodes $s_{1}=\min \mathcal{I}_{1}, \ldots, s_{k_{1}}=\min \mathcal{I}_{k_{1}}$ (otherwise $\mathcal{I} \in \mathcal{S}_{1}$ ). Since there are $k_{1} 2^{m_{2}-m_{1}}$ nodes on the level $m_{2}$ which are followers of the nodes $s_{1}, \ldots, s_{k_{1}}$, we deduce that $k_{2} \leq$ $2^{m_{2}}-k_{1} 2^{m_{2}-m_{1}}$. As previously, we set $\mathcal{S}_{2}=\left\{\mathcal{J} \in \mathcal{S} \mid \mathcal{J} \notin \mathcal{S}_{1}\right.$, and there is $\mathcal{I} \in \mathcal{S} \backslash \mathcal{S}_{1}$ such that $\operatorname{lev}(\min \mathcal{I})=m_{2}$ and $\left.\min \mathcal{I} \leq \min \mathcal{J}\right\}$. Then we have

$$
\sum_{\mathcal{I} \in \mathcal{S}_{2}}\left|\mathcal{I}^{*}(x)\right|^{2} \leq \frac{k_{2}}{2^{m_{2}}} 3(\sqrt{2}+1)^{2} .
$$

Repeating the same argument, after finitely many steps, we exhaust all the segments of the family $\mathcal{S}$. Let us describe what we have at the last step. We set $m_{l}=\min \left\{\operatorname{lev}(\min \mathcal{I}) \mid \mathcal{I} \in \mathcal{S} \backslash\left(\mathcal{S}_{1} \cup \cdots \cup \mathcal{S}_{l-1}\right)\right\}$. We 
also assume that there are $k_{l}$ segments $\mathcal{I}$ such that $\mathcal{I} \in \mathcal{S} \backslash\left(\mathcal{S}_{1} \cup \cdots \cup\right.$ $\left.\mathcal{S}_{l-1}\right)$ and $\operatorname{lev}(\min \mathcal{I})=m_{l}$. Then we observe that

$$
k_{l} \leq 2^{m_{l}}-k_{1} 2^{m_{l}-m_{1}}-\ldots-k_{l-1} 2^{m_{l}-m_{l-1}} .
$$

Therefore,

$$
\frac{k_{1}}{2^{m_{1}}}+\frac{k_{2}}{2^{m_{2}}}+\ldots+\frac{k_{l}}{2^{m_{l}}} \leq 1 .
$$

Finally, we set $\mathcal{S}_{l}=\left\{\mathcal{J} \in \mathcal{S} \mid \mathcal{J} \notin \mathcal{S}_{1} \cup \cdots \cup \mathcal{S}_{l-1}\right.$, and there is $\mathcal{I} \in \mathcal{S} \backslash \cup_{k=1}^{l-1} \mathcal{S}_{k}$ such that $\operatorname{lev}(\min \mathcal{I})=m_{l}$ and $\left.\min \mathcal{I} \leq \min \mathcal{J}\right\}$, and we have

$$
\sum_{\mathcal{I} \in \mathcal{S}_{l}}\left|\mathcal{I}^{*}(x)\right|^{2} \leq \frac{k_{l}}{2^{m_{l}}} 3(\sqrt{2}+1)^{2} .
$$

It is easy now to estimate the $\operatorname{sum} \sum_{\mathcal{I} \in \mathcal{S}}\left|\mathcal{I}^{*}(x)\right|^{2}$. Indeed,

$$
\begin{aligned}
\sum_{\mathcal{I} \in \mathcal{S}}\left|\mathcal{I}^{*}(x)\right|^{2} & =\sum_{\mathcal{I} \in \mathcal{S}_{1}}\left|\mathcal{I}^{*}(x)\right|^{2}+\cdots+\sum_{\mathcal{I} \in \mathcal{S}_{l}}\left|\mathcal{I}^{*}(x)\right|^{2} \\
& \leq \frac{k_{1}}{2^{m_{1}}} 3(\sqrt{2}+1)^{2}+\cdots+\frac{k_{l}}{2^{m_{l}}} 3(\sqrt{2}+1)^{2} \\
& \leq 3(\sqrt{2}+1)^{2}\left[\frac{k_{1}}{2^{m_{1}}}+\cdots+\frac{k_{l}}{2^{m_{l}}}\right] \\
& \leq 3(\sqrt{2}+1)^{2},
\end{aligned}
$$

and the proof of the claim is complete.

To finish the proof of the theorem we need only observe that, for any $n \in \mathbb{N}$ and any $t_{1}, \ldots, t_{n} \in \mathbb{R}$, we have

$$
\left\|\sum_{i=1}^{n} t_{i} x_{i}\right\| \leq \max _{1 \leq i \leq n}\left|t_{i}\right|\left\|\sum_{i=1}^{n} x_{i}\right\| .
$$

Indeed, if we set $u=\sum_{i=1}^{n} t_{i} x_{i}$ and $x=\sum_{i=1}^{n} x_{i}$, then, for any node $s \in \mathcal{D}$, we obtain

$$
u(s)=\sum_{i=1}^{n} t_{i} x_{i}(s)=t_{\operatorname{lev}(s)} \frac{1}{2^{\operatorname{lev}(s) / 2}} .
$$


Thus, for any segment $\mathcal{I}$,

$$
\begin{aligned}
\left|\mathcal{I}^{*}(u)\right| & \leq \sum_{s \in \mathcal{I}}|u(s)|=\sum_{s \in \mathcal{I}}\left|t_{\operatorname{lev}(s)}\right| \frac{1}{2^{\operatorname{lev}(s) / 2}} \\
& \leq \max _{1 \leq i \leq n}\left|t_{i}\right| \sum_{s \in \mathcal{I}} \frac{1}{2^{\operatorname{lev}(s) / 2}} \\
& =\max _{1 \leq i \leq n}\left|t_{i}\right|\left|\mathcal{I}^{*}(x)\right| .
\end{aligned}
$$

Hence, for any admissible family $\mathcal{S}$,

$$
\sum_{\mathcal{I} \in \mathcal{S}}\left|\mathcal{I}^{*}(u)\right|^{2} \leq\left(\max \left|t_{i}\right|\right)^{2} \sum_{\mathcal{I} \in \mathcal{S}}\left|\mathcal{I}^{*}(x)\right|^{2}
$$

and the result follows. Therefore, using the previous claim, we have

$$
\left\|\sum_{i=1}^{n} t_{i} x_{i}\right\| \leq \sqrt{3}(\sqrt{2}+1) \max _{1 \leq i \leq n}\left|t_{i}\right|,
$$

that is, the sequence $\left(x_{i}\right)$ is equivalent to the usual basis of $c_{0}$.

We now turn our attention to the Opial condition. As the space $T F$ indicates, the embedding of $c_{0}$ in a Banach space does not imply that the latter does not satisfy the Opial condition. However, in our case, it seems that it is the presence of $c_{0}$ which causes $X$ to fail the Opial property.

In the following, rather than showing that $X$ does not satisfy the Opial condition, we prove a stronger result. Recall that a nonempty closed bounded convex subset $K$ of a Banach space is said to have normal structure if each closed, bounded, convex subset $C$ of $K$ with at least two points contains a non-diametral point, that is, there exists $x_{0} \in C$ such that $\sup \left\{\left\|x_{0}-x\right\| \mid x \in C\right\}<\operatorname{diam}(C)$. A Banach space is said to have weak normal structure if every weakly compact, convex subset $K$ with diam $(K)>0$ has normal structure.

Theorem 5.5. The space $X$ does not possess weak normal structure.

Proof. According to a characterization of normal structure, it suffices to show that $X$ contains a weakly null diametral sequence $\left(y_{n}\right)$. Recall that a bounded sequence $\left(y_{n}\right)$ is said to be diametral if it is 
nonconstant and

$$
\lim _{n \rightarrow \infty} d\left(y_{n}, \operatorname{co}\left\{y_{0}, \ldots, y_{n-1}\right\}\right)=\operatorname{diam}\left(\left\{y_{n}\right\}\right)
$$

where $d\left(y_{n}, \operatorname{co}\left\{y_{0}, \ldots, y_{n-1}\right\}\right)=\inf \left\{\left\|y_{n}-y\right\| \mid y \in \operatorname{co}\left\{y_{0}, \ldots, y_{n-1}\right\}\right\}$. Once the sequence $\left(y_{n}\right)$ has been constructed, then we have that the set $K=\overline{\mathrm{co}}\left\{y_{n} \mid n \in \mathbb{N}\right\}$ is a weakly compact convex set which does not have normal structure.

Since the standard example of a diametral sequence is the usual basis of $c_{0}$, our first thought is to examine the sequence $\left(x_{n}\right)$ given in Theorem 5.3. An easy estimate, though, implies that $\left(x_{n}\right)$ is not diametral. However, a block basis of $\left(x_{n}\right)$ solves our problem.

Indeed, we first consider a sequence $k_{0}=0<k_{1}<k_{2}<\cdots$ of levels of the tree $\mathcal{D}$ such that, for each $n \in \mathbb{N}$,

$$
\frac{1}{(\sqrt{2})^{k_{n}}}+\frac{1}{(\sqrt{2})^{k_{n}+1}}<\frac{1}{(\sqrt{2})^{k_{n-1}+1}} .
$$

Then we set $y_{n}=x_{k_{n}}+x_{k_{n}+1}$. Since $\left(x_{n}\right)$ is weakly null, we have that $\left(y_{n}\right)$ also converges weakly to zero. We next show that $\left(y_{n}\right)$ is a diametral sequence.

We first estimate the norm of $y_{n}$. Observe that, for each $n$, the support of the vector $y_{n}$ consists of the nodes $s$ on the level $k_{n}$, where $y_{n}(s)=1 /\left[(\sqrt{2})^{k_{n}}\right]$, and of the nodes $s$ on the level $k_{n}+1$, where $y_{n}(s)=1 /\left[(\sqrt{2})^{k_{n}+1}\right]$. Let $\mathcal{S}$ be an admissible family. Then there is no point in choosing the segments $\mathcal{I} \in \mathcal{S}$ so that lev $(\min \mathcal{I})<k_{n}$. Thus, the best choice for $\mathcal{S}$ is the following. $\mathcal{S}$ consists of the segments $\mathcal{I}_{s}=\{s, s \frown 0\}$ and the segments $\mathcal{J}_{s}=\{s \frown 1\}$, where $s$ varies over the nodes of the level $k_{n}$. Hence, we have

$$
\begin{aligned}
\left\|y_{n}\right\|^{2} & =\sum_{i=1}^{2^{k_{n}}}\left[\left|\mathcal{I}_{i}^{*}\left(y_{n}\right)\right|^{2}+\left|\mathcal{J}_{i}^{*}\left(y_{n}\right)\right|^{2}\right] \\
& =\sum_{i=1}^{2^{k_{n}}}\left[\left(\frac{1}{(\sqrt{2})^{k_{n}}}+\frac{1}{(\sqrt{2})^{k_{n}+1}}\right)^{2}+\left(\frac{1}{(\sqrt{2})^{k_{n}+1}}\right)^{2}\right] \\
& =2+\sqrt{2} .
\end{aligned}
$$

Therefore, $\left\|y_{n}\right\|=\sqrt{2+\sqrt{2}}$ for each $n \in \mathbb{N}$. Now let $y$ be any vector belonging to co $\left\{y_{0}, \ldots, y_{n-1}\right\}$. Then $y$ and $y_{n}$ have disjoint supports. 
Hence, it is easy to see that $\left\|y_{n}-y\right\| \geq\left\|y_{n}\right\|=\sqrt{2+\sqrt{2}}$. Therefore, $d\left(y_{n}, \operatorname{co}\left\{y_{0}, \ldots, y_{n-1}\right\}\right) \geq \sqrt{2+\sqrt{2}}$ for each $n \in \mathbb{N}$, which implies that

$$
\lim _{n \rightarrow \infty} d\left(y_{n}, \operatorname{co}\left\{y_{0}, \ldots, y_{n-1}\right\}\right) \geq \sqrt{2+\sqrt{2}} .
$$

We next argue that, for any $n, m \in \mathbb{N}, n<m$, we have $\left\|y_{n}-y_{m}\right\|=$ $\sqrt{2+\sqrt{2}}$. For our convenience, let us set $k_{n}=k, k_{m}=l$ and $x=y_{n}-y_{m}$. The choice of the sequence $\left(k_{n}\right)$ implies that

$$
\frac{1}{(\sqrt{2})^{l}}+\frac{1}{(\sqrt{2})^{l+1}}<\frac{1}{(\sqrt{2})^{k+1}} .
$$

Observe that the support of the vector $x$ consists of the nodes on the levels $k$ and $k+1$, where the coordinates of $x$ are positive, and of the nodes on the levels $l$ and $l+1$, where the coordinates $x(s)$ are negative. Let $\mathcal{S}$ be an admissible family on the dyadic tree. We search for that $\mathcal{S}$ which maximizes the sum $\sum_{\mathcal{I} \in \mathcal{S}}\left|\mathcal{I}^{*}(x)\right|^{2}$. First, let us assume that the segments $\mathcal{I}$ of $\mathcal{S}$ intersect only the support of $y_{n}$ (respectively, $y_{m}$ ). Then we have $\sum_{\mathcal{I} \in \mathcal{S}}\left|\mathcal{I}^{*}(x)\right|^{2} \leq\left\|y_{n}\right\|^{2}$ (respectively, $\left.\left\|y_{m}\right\|^{2}\right)=2+\sqrt{2}$.

Let us now assume that there are segments $\mathcal{I} \in \mathcal{S}$ which intersect both the supports of $y_{n}$ and $y_{m}$. Since the coordinates $x(s)$, where $\operatorname{lev}(s)=l$ or $l+1$, are negative, if we set $\mathcal{J}=\mathcal{I} \cap\{s \mid \operatorname{lev}(s)=$ $k$ or $k+1\}$ then (using the inequality (5.4)) we have $\left|\mathcal{I}^{*}(x)\right| \leq\left|\mathcal{J}^{*}(x)\right|$. Therefore, the family $\mathcal{S}$ that we are looking for cannot contain such segments $\mathcal{I}$, since any of these segments can be replaced by its intersection with the levels $k$ and $k+1$.

There is only one possibility for the family $\mathcal{S}$ which remains open. The case where $\mathcal{S}$ contains segments which intersect only the support of $y_{n}$, and at the same time $\mathcal{S}$ contains segments which intersect only the support of $y_{m}$. Let $s$ be any node on the level $k$. Then we may assume that $\mathcal{I}_{s}=\{s, s \frown 0\}$ belongs to $\mathcal{S}$. Since $\mathcal{S}$ is admissible, we are allowed to choose only one segment whose minimum node is a follower of $s$. By the inequality (5.4), the best choice for us is to consider the segment $\mathcal{J}_{s}=\{s \frown 1\}$. Hence, the family $\mathcal{S}$ which maximizes the sum $\sum_{\mathcal{I} \in \mathcal{S}}\left|\mathcal{I}^{*}(x)\right|^{2}$ is $\mathcal{S}=\left\{\mathcal{I}_{s}, \mathcal{J}_{s}\right\}_{s: \operatorname{lev}(s)=k}$, which is exactly the family giving the norm of $\left\|y_{n}\right\|$. Thus, $\|x\|=\left\|y_{n}\right\|=\sqrt{2+\sqrt{2}}$. (We can also consider the family $\mathcal{S}$ which contains all segments $\{s \frown 0\}$ and $\{s \frown 1\}$, where lev $(s)=k$, each one of which is combined with one and only one 
segment intersecting the support of $y_{m}$. However, a simple estimation shows that this is not the best choice for $\mathcal{S}$.)

Finally, for any $n \neq m$, we have $\left\|y_{n}-y_{m}\right\|=\sqrt{2+\sqrt{2}}$, and hence $\operatorname{diam}\left(\left\{y_{n}\right\}\right)=\sup _{n, m}\left\|y_{n}-y_{m}\right\|=\sqrt{2+\sqrt{2}}$. Consequently,

$$
\operatorname{diam}\left(\left\{y_{n}\right\}\right) \leq \lim _{n \rightarrow \infty} d\left(y_{n}, \operatorname{co}\left\{y_{0}, \ldots, y_{n-1}\right\}\right) .
$$

The reverse inequality is obvious. Thus, $\left(y_{n}\right)$ is a weakly null diametral sequence.

It is well known (see [5]) that the Opial condition implies that the space has weak normal structure. Hence, by the above theorem, we immediately get the following.

Corollary 5.6. The space $X$ does not satisfy the Opial condition.

Finally, we observe that Theorem 5.3 provides us with a lot of subspaces isomorphic to $c_{0}$, and Theorem 5.5 provides us with a lot of weakly compact convex subsets which do not possess normal structure. This will become clear as soon as the next proposition is proved. Recall that a partially ordered set $\mathcal{T}$ is called a dyadic tree if it is order isomorphic to $(\mathcal{D}, \leq)$. If $\mathcal{T}$ is a dyadic tree and $\mathcal{T} \subseteq \mathcal{D}$, then we say that $\mathcal{T}$ is a subtree of $\mathcal{D}$.

Proposition 5.7. For any subtree $\mathcal{T}$ of $\mathcal{D}$, the space $\overline{\operatorname{span}}\left\{e_{s} \mid s \in \mathcal{T}\right\}$ is isometric to $X$.

Proof. Suppose that $\varphi: \mathcal{D} \rightarrow \mathcal{T}$ is an order isomorphism. We need only show that, for any finitely supported sequence $\left(\lambda_{s}\right)_{s \in \mathcal{D}}$ of scalars, we have

$$
\left\|\sum_{s \in \mathcal{D}} \lambda_{s} e_{s}\right\|=\left\|\sum_{s \in \mathcal{D}} \lambda_{s} e_{\varphi(s)}\right\| .
$$

Let us write $x=\sum_{s \in \mathcal{D}} \lambda_{s} e_{s}$ and $y=\sum_{s \in \mathcal{D}} \lambda_{s} e_{\varphi(s)}$. By the definition of the norm, there is an admissible family $\mathcal{S}=\left\{\mathcal{I}_{i}\right\}_{i=1}^{r}$ of segments of $\mathcal{D}$ such that

$$
\|x\|=\left(\sum_{i=1}^{r}\left|\mathcal{I}_{i}^{*}(x)\right|^{2}\right)^{1 / 2} .
$$


For any $i=1,2, \ldots, r$, we set $\mathcal{J}_{i}=\left\{s \in \mathcal{D} \mid \varphi\left(\min \mathcal{I}_{i}\right) \leq s \leq\right.$ $\left.\varphi\left(\max \mathcal{I}_{i}\right)\right\}$. Clearly, $\left\{\mathcal{J}_{i}\right\}_{i=1}^{r}$ are pairwise disjoint segments of $\mathcal{D}$, and we have $\mathcal{J}_{i}^{*}(y)=\mathcal{I}_{i}^{*}(x)$. Further, the family $\left\{\mathcal{J}_{i}\right\}_{i=1}^{r}$ is admissible. Indeed, if this is not true, then there are three segments, say the segments $\mathcal{J}_{1}, \mathcal{J}_{2}$ and $\mathcal{J}_{3}$, so that $\min \mathcal{J}_{1}<\min \mathcal{J}_{2}$ and $\min \mathcal{J}_{1}<\min \mathcal{J}_{3}$, that is, $\varphi\left(\min \mathcal{I}_{1}\right)<\varphi\left(\min \mathcal{I}_{2}\right)$ and $\varphi\left(\min \mathcal{I}_{1}\right)<\varphi\left(\min \mathcal{I}_{3}\right)$. Since $\varphi$ is an order isomorphism, we obtain $\min \mathcal{I}_{1}<\min \mathcal{I}_{2}$ and $\min \mathcal{I}_{1}<\min \mathcal{I}_{3}$. Hence, $\mathcal{S}$ is not admissible, and we have reached a contradiction. Therefore, $\left\{\mathcal{J}_{i}\right\}_{i=1}^{r}$ is admissible, and thus

$$
\|x\|=\left(\sum_{i=1}^{r}\left|\mathcal{J}_{i}^{*}(y)\right|^{2}\right)^{1 / 2} \leq\|y\| .
$$

Consider now an admissible family $\mathcal{S}=\left\{\mathcal{J}_{i}\right\}_{i=1}^{r}$ of segments of $\mathcal{D}$ such that $\|y\|=\left(\sum_{i=1}^{r}\left|\mathcal{J}_{i}^{*}(y)\right|^{2}\right)^{1 / 2}$. For any $i=1,2, \ldots, r$, we set $\mathcal{I}_{i}=\left\{s \in \mathcal{D} \mid \varphi(s) \in \mathcal{J}_{i}\right\}$. Then, $\left\{\mathcal{I}_{i}\right\}_{i=1}^{r}$ are pairwise disjoint segments, and we have $\mathcal{I}_{i}^{*}(x)=\mathcal{J}_{i}^{*}(y)$. Further, if we assume that $\left\{\mathcal{I}_{i}\right\}_{i=1}^{r}$ is not admissible, then there are three segments $\mathcal{I}_{1}, \mathcal{I}_{2}$ and $\mathcal{I}_{3}$ with $s_{1}=\min \mathcal{I}_{1}<s_{2}=\min \mathcal{I}_{2}$ and $s_{1}<s_{3}=\min \mathcal{I}_{3}$. Hence, $\varphi\left(s_{1}\right)<\varphi\left(s_{2}\right)$ and $\varphi\left(s_{1}\right)<\varphi\left(s_{3}\right)$. However, min $\mathcal{J}_{i} \leq \varphi\left(s_{i}\right)$, and thus $\min \mathcal{J}_{1}<\min \mathcal{J}_{2}, \min \mathcal{J}_{1}<\min \mathcal{J}_{3}$ which is a contradiction. Therefore, $\left\{\mathcal{I}_{i}\right\}_{i=1}^{r}$ is admissible and

$$
\|y\|=\left(\sum_{i=1}^{r}\left|\mathcal{I}_{i}^{*}(x)\right|^{2}\right)^{1 / 2} \leq\|x\| .
$$

6. The second space. In this section we introduce a second treelike Banach space and we show that it shares similar properties to the space $X$ studied in the previous sections. The new space, which will be denoted by $Y$, follows from a modification in the notion of admissibility.

Let $\mathcal{S}$ be a finite family of pairwise disjoint segments. Throughout this section, $\mathcal{S}$ is called admissible whenever the following condition holds: for any segment $\mathcal{I} \in \mathcal{S}$, if we set $\mathcal{S}_{\mathcal{I}}=\{\mathcal{J} \in \mathcal{S} \mid \min \mathcal{I} \leq$ $\min \mathcal{J}\}$, then the minimum nodes of the segments $\mathcal{J} \in \mathcal{S}_{\mathcal{I}}$ are pairwise comparable. The space $Y$ is the completion of $c_{00}(\mathcal{D})$ with respect to 
the norm defined as follows: for any $x \in c_{00}(\mathcal{D})$,

$$
\|x\|=\sup \left(\sum_{\mathcal{I} \in \mathcal{S}}\left|\mathcal{I}^{*}(x)\right|^{2}\right)^{1 / 2}
$$

where the supremum is taken over all finite admissible (in the previous sense) families $\mathcal{S}$ of pairwise disjoint segments.

Some remarks on the notion of admissibility follow. First, loosely speaking, suppose that we want to construct an admissible family, and let us start with any segment $\mathcal{I}$. Then we can consider as many segments $\mathcal{J}$ as we want such that $\min \mathcal{I}<\min \mathcal{J}$, provided that the minimum nodes of these segments lie on the same branch. (On the contrary, in Section 3, we were obliged to choose at most one segment $\mathcal{J}$ with $\min \mathcal{I}<\min \mathcal{J}$.) Note that, if we were allowed to consider pairwise disjoint segments $\mathcal{J}$ with $\min \mathcal{I}<\min \mathcal{J}$ without any other restriction, then we would simply obtain the space $J T$.

Secondly, suppose that $\mathcal{S}$ is an admissible family of pairwise disjoint segments and $\mathcal{S}^{\prime}$ a family of segments such that for any $\mathcal{I}^{\prime} \in \mathcal{S}^{\prime}$ there is $\mathcal{I} \in \mathcal{S}$ with $\mathcal{I}^{\prime} \subseteq \mathcal{I}$. Then $\mathcal{S}^{\prime}$ need not be admissible. For example, let $\mathcal{S}=\left\{\mathcal{I}_{0}, \mathcal{I}_{1}, \mathcal{I}_{2}\right\}$ where $\mathcal{I}_{i}, i=0,1,2$, are segments such that $\min \mathcal{I}_{0}=\emptyset, \min \mathcal{I}_{1}=\{(1)\}$ and $\min \mathcal{I}_{2}=\{(1,1)\}$. Clearly, $\mathcal{S}$ is admissible. However, if $\mathcal{I}_{1}$ is replaced by $\mathcal{I}_{1}^{\prime}=\mathcal{I}_{1} \backslash\left\{\min \mathcal{I}_{1}\right\}$, then the family $\mathcal{S}^{\prime}=\left\{\mathcal{I}_{0}, \mathcal{I}_{1}^{\prime}, \mathcal{I}_{2}\right\}$ is not admissible. This situation brings about some difficulty in the study of the space $Y$. Any time we want to cut the segments of an admissible family we have to verify that the new family obtained is also admissible. Nevertheless, the previous example indicates actually the only case which causes some problems. For instance it is not hard to see that if $\mathcal{S}$ is admissible and $m$ is any level then both $\mathcal{S}^{\prime}=\{\mathcal{I} \cap\{s: \operatorname{lev}(s) \leq m\} \mid \mathcal{I} \in \mathcal{S}\}$ and $\mathcal{S}^{\prime \prime}=\{\mathcal{I} \cap\{s: \operatorname{lev}(s) \geq m\} \mid \mathcal{I} \in \mathcal{S}\}$ are admissible families.

The space $Y$ has similar properties to the space $X$. First, we observe that $Y$ is separable, with non-separable dual, and it does not contain an isomorphic copy of $\ell_{1}$. The latter follows from the fact that Theorem 3.2 remains valid for the space $Y$.

\section{Theorem 6.1.}

(i) The quotient space $Y^{*} / F$ is isomorphic to $\ell_{2}(\Gamma)$ (where $F$ is the space generated by the biorthogonal functionals $\left.e_{s}^{*}, s \in \mathcal{D}\right)$. 
(ii) The second dual $Y^{* *}$ is isomorphic to $F^{*} \oplus \ell_{2}(\Gamma)$.

Further, we can prove the following.

Theorem 6.2. The space $Y$ has the fixed point property.

The proof is the same as the proof of Theorem 4.1.

One basic difference between $Y$ and $X$ is detected in the structure of their subspaces. More precisely, the analogue of Proposition 3.4 for the space $Y$ is the following.

\section{Proposition 6.3.}

(i) For any chain $\left(s_{n}\right)$ of $\mathcal{D}$, the space $\overline{\operatorname{span}}\left\{e_{s_{n}} \mid n \in \mathbb{N}\right\}$ is isometrically isomorphic to the James quasi-reflexive space $J$.

(ii) For any antichain $\left(s_{n}\right)$ of $\mathcal{D}$, the space $\overline{\operatorname{span}}\left\{e_{s_{n}} \mid n \in \mathbb{N}\right\}$ is isometrically isomorphic to $\ell_{2}$.

That is, for any branch $B$, the space generated by the vectors $e_{s}$, $s \in B$, is not isomorphic to $c_{0}$, but it is isomorphic to the space $J$. Nevertheless, the analogue of Theorem 5.3 for the space $Y$ remains valid from which we deduce that $Y$ also contains isomorphic copies of $c_{0}$.

Theorem 6.4. Suppose that $\left(x_{n}\right)$ is the sequence of Theorem 5.3, that is, $x_{n}(s)=1 / 2^{n / 2}$ if lev $(s)=n$ and $x_{n}(s)=0$ otherwise. Then $\left(x_{n}\right)$ in the space $Y$ is equivalent to the usual basis of $c_{0}$. In particular, for any $n \in \mathbb{N}$ and any $t_{1}, \ldots, t_{n} \in \mathbb{R}$, we have

$$
\max _{1 \leq i \leq n}\left|t_{i}\right| \leq\left\|\sum_{i=1}^{n} t_{i} x_{i}\right\| \leq 2(\sqrt{2}+1) \max _{1 \leq i \leq n}\left|t_{i}\right| .
$$

Finally, we do not know whether the space $Y$ has normal structure or not. However, we can prove the next result.

Theorem 6.5. The space $Y$ does not satisfy the Opial condition. 
Proof. We first consider the sequence $\left(x_{n}\right)$ as in the previous theorem. It is easy to see that $\left\|x_{n}\right\|=1$ for any $n \in \mathbb{N}$ and, therefore, $\lim _{n \rightarrow \infty}\left\|x_{n}\right\|=1$. Further, by Corollary 3.3 (which also remains valid for the space $Y$ ), it follows that $\left(x_{n}\right)$ converges weakly to zero. In order to prove the theorem we need to find a vector $x \in Y, x \neq 0$, such that $\lim _{n \rightarrow \infty}\left\|x_{n}+x\right\|=1$. For this reason, we set $x=a e_{\emptyset}$ where $a$ is any real number with $0<|a| \leq 1$ and we show that $x$ has the desired property.

Indeed, fix a positive integer $n$, and let $\mathcal{S}=\left\{\mathcal{I}_{i}\right\}_{i=1}^{r}$ be an admissible family of pairwise disjoint segments. In order to estimate the norm $\left\|x_{n}+x\right\|$, we search for the family $\mathcal{S}$ which maximizes the sum

$$
\left(\sum_{i=1}^{r}\left|\mathcal{I}_{i}^{*}\left(x_{n}+x\right)\right|^{2}\right)^{1 / 2}
$$

We distinguish two cases.

Case 1. Suppose that $\emptyset \notin \cup_{i=1}^{r} \mathcal{I}_{i}$. Then we can consider a family $\mathcal{S}$ such that any node $s$ with $\operatorname{lev}(s)=n$ belongs to some segment $\mathcal{I} \in \mathcal{S}$. This turns out to be the best choice for $\mathcal{S}$ in this case. For instance, if $\{0,1\}^{n}=\left\{s_{1}, \ldots, s_{2^{n}}\right\}$ is an enumeration of the nodes on level $n$, then we set $\mathcal{S}=\left\{\mathcal{I}_{i}\right\}_{i=1}^{2^{n}}$, where $\mathcal{I}_{i}=\left\{s_{i}\right\}$, and we have

$$
\left(\sum_{i=1}^{2^{n}}\left|\mathcal{I}_{i}^{*}\left(x_{n}+x\right)\right|^{2}\right)^{1 / 2}=\left(\sum_{i=1}^{2^{n}}\left(\frac{1}{2^{n / 2}}\right)^{2}\right)^{1 / 2}=1
$$

Case 2. Assume now that $\emptyset$ belongs to some segment of the family $\mathcal{S}$, and say that $\emptyset \in \mathcal{I}_{1}$. Then $\min \mathcal{I}_{1} \leq \min \mathcal{I}_{i}$ for every $i=1,2, \ldots, r$. Since $\mathcal{S}$ is admissible, it follows that the minimum nodes of the segments $\left\{\mathcal{I}_{i}\right\}_{i=1}^{r}$ lie on the same branch. Therefore, there are at most $n+1$ segments of $\mathcal{S}$ which intersect the level $n$, and hence at most $n+1$ nodes on level $n$ belong to the union $\cup_{i=1}^{r} \mathcal{I}_{i}$. Consequently, in this case, the best choice for us is to consider a family $\mathcal{S}=\left\{\mathcal{I}_{i}\right\}_{i=1}^{n+1}$ such that $\emptyset \in \mathcal{I}_{1}, \min \mathcal{I}_{i+1}$ is an immediate follower of $\min \mathcal{I}_{i}$ and each $\mathcal{I}_{i}$ 
intersects the level $n$. Then we have

$$
\begin{aligned}
\left(\sum_{i=1}^{n+1}\left|\mathcal{I}_{i}^{*}\left(x_{n}+x\right)\right|^{2}\right)^{1 / 2} & =\left(\left|\mathcal{I}_{1}^{*}\left(x_{n}+x\right)\right|^{2}+\sum_{i=2}^{n+1}\left|\mathcal{I}_{i}^{*}\left(x_{n}+x\right)\right|^{2}\right)^{1 / 2} \\
& =\left[\left(a+\frac{1}{2^{n / 2}}\right)^{2}+\frac{n}{2^{n}}\right]^{1 / 2} .
\end{aligned}
$$

Therefore,

$$
\left\|x_{n}+x\right\|=\max \left\{1,\left[\left(a+\frac{1}{2^{n / 2}}\right)^{2}+\frac{n}{2^{n}}\right]^{1 / 2}\right\} .
$$

Since

$$
\left[\left(a+\frac{1}{2^{n / 2}}\right)^{2}+\frac{n}{2^{n}}\right]^{1 / 2} \longrightarrow|a| \leq 1,
$$

we deduce that $\lim _{n \rightarrow \infty}\left\|x_{n}+x\right\|=1$, and the proof is complete.

\section{Further remarks.}

Remark 7.1. In Section 5 (respectively, 6) we proved that the space $X$ (respectively, $Y$ ) does not satisfy the Opial condition. In the following, we strengthen this result by showing that any closed finite codimensional subspace of $X$ (respectively, $Y$ ) fails the Opial property.

Theorem 7.2. Suppose that $Z$ is a closed, finite codimensional subspace of $X$ (respectively, $Y$ ). Then $Z$ does not satisfy the Opial condition.

Proof. We describe the proof in the case of the space $X$. The necessary changes for the space $Y$ can be completed easily. We also assume that $Z$ is a subspace of $X$ which has codimension one. The general case of finite codimensional subspaces is similar.

Since $Z$ has codimension one, there is $x_{0} \in X$ with $\left\|x_{0}\right\|=1$, such that $X=Z \oplus\left\langle x_{0}\right\rangle$. Therefore, any $x \in X$ can be written uniquely in the form $x=z+\lambda x_{0}$, where $z \in Z$ and $\lambda \in \mathbb{R}$.

Consider now the sequence $\left(x_{n}\right)$ of Theorem 5.3, that is, $x_{n}(s)=$ $1 / 2^{n / 2}$ if lev $(s)=n$ and $x_{n}(s)=0$, otherwise. We know that $\left\|x_{n}\right\|=1$ and $\left(x_{n}\right)$ converges weakly to zero. For every $n \in \mathbb{N}$, the vector $x_{n}$ is 
written in the form $x_{n}=z_{n}+\lambda_{n} x_{0}$. Observe that $\left(\lambda_{n}\right)$ is a bounded sequence. Therefore, by passing to a subsequence, if necessary, we may assume that $\lambda_{n} \rightarrow \lambda \in \mathbb{R}$. Then, for each $x^{*} \in X^{*}$, we have

$$
x^{*}\left(z_{n}+\lambda x_{0}\right)=x^{*}\left(x_{n}\right)+\left(\lambda-\lambda_{n}\right) x^{*}\left(x_{0}\right),
$$

and hence, $x^{*}\left(z_{n}+\lambda x_{0}\right) \rightarrow 0$, that is, $\left(z_{n}\right)$ converges weakly to $-\lambda x_{0}$. Since $Z$ is closed, we deduce that $-\lambda x_{0}$ belongs to $Z$. However, this can happen if and only if $\lambda=0$. Therefore, $\lambda_{n} \rightarrow 0$, and $\left(z_{n}\right)$ is a weakly null sequence. Further, by the equation $z_{n}=x_{n}-\lambda_{n} x_{0}$, it follows

$$
\left\|x_{n}\right\|-\left|\lambda_{n}\right|\left\|x_{0}\right\| \leq\left\|z_{n}\right\| \leq\left\|x_{n}\right\|+\left|\lambda_{n}\right|\left\|x_{0}\right\|,
$$

which implies that $\lim _{n \rightarrow \infty}\left\|z_{n}\right\|=1$.

Consider now any nonzero element $z_{0} \in Z$ whose support is finite. Let $m$ be a level such that $P_{m} z_{0}=0$. By multiplication with a suitable constant, we also have $\left\|z_{0}\right\| \leq 1 /\left(2^{(m+1) / 2}\right)$. To complete the proof of the theorem we need to show that $\lim _{n \rightarrow \infty}\left\|z_{n}-z_{0}\right\|=\lim _{n \rightarrow \infty}\left\|z_{n}\right\|=$ 1.

Fix any integer $n>m$. In order to estimate the norm $\left\|z_{n}-z_{0}\right\|$ we consider an admissible family $\left\{\mathcal{I}_{i}\right\}_{i=1}^{r}$ of pairwise disjoint segments, and we distinguish the following cases.

Case 1 . Suppose that $\operatorname{lev}\left(\min \mathcal{I}_{i}\right) \geq m$ for any $i=1,2, \ldots, r$. Then $\mathcal{I}_{i}^{*}\left(z_{0}\right)=0$, and therefore,

$$
\left(\sum_{i=1}^{r}\left|\mathcal{I}_{i}^{*}\left(z_{n}-z_{0}\right)\right|^{2}\right)^{1 / 2}=\left(\sum_{i=1}^{r}\left|\mathcal{I}_{i}^{*}\left(z_{n}\right)\right|^{2}\right)^{1 / 2} \leq\left\|z_{n}\right\| .
$$

Case 2. Assume now that there is at least one segment, say the segment $\mathcal{I}_{1}$, such that lev $\left(\min \mathcal{I}_{1}\right)<m$. Let us also set $s_{1}=\min \mathcal{I}_{1}$ and $k=\operatorname{lev}\left(\min \mathcal{I}_{1}\right)<m$. Observe that there are $2^{n-k}$ nodes on the level $n$ which are followers of the node $s_{1}$. Further, since $\mathcal{S}$ is admissible, at most two of these $2^{n-k}$ nodes may belong to the union $\cup_{i=1}^{r} \mathcal{I}_{i}$. So, if we set

$$
\begin{aligned}
& A_{1}=\left\{i \mid \operatorname{lev}\left(\max \mathcal{I}_{i}\right)<m\right\} \\
& A_{2}=\left\{i \mid \operatorname{lev}\left(\min \mathcal{I}_{i}\right) \geq m\right\} \\
& A_{3}=\left\{i \mid \operatorname{lev}\left(\min \mathcal{I}_{i}\right)<m \leq \operatorname{lev}\left(\max \mathcal{I}_{i}\right)\right\},
\end{aligned}
$$


then there are at most $2^{n}-2^{n-k}+1$ nodes on the level $n$ which belong to $\cup_{i \in A_{2}} \mathcal{I}_{i}$. We also observe that the cardinality of the set $A_{3}$ is less or equal to $2^{m}$. Now we have

$$
\begin{aligned}
& \sum_{i \in A_{1}}\left|\mathcal{I}_{i}^{*}\left(z_{n}-z_{0}\right)\right|^{2}=\sum_{i \in A_{1}}\left|\mathcal{I}_{i}^{*}\left(x_{n}\right)+\mathcal{I}_{i}^{*}\left(-\lambda_{n} x_{0}\right)-\mathcal{I}_{i}^{*}\left(z_{0}\right)\right|^{2} \\
&=\sum_{i \in A_{1}}\left|\mathcal{I}_{i}^{*}\left(-\lambda_{n} x_{0}\right)-\mathcal{I}_{i}^{*}\left(z_{0}\right)\right|^{2} \\
& \leq\left[\left(\sum_{i \in A_{1}}\left|\mathcal{I}_{i}^{*}\left(-\lambda_{n} x_{0}\right)\right|^{2}\right)^{1 / 2}+\left(\sum_{i \in A_{1}}\left|\mathcal{I}_{i}^{*}\left(z_{0}\right)\right|^{2}\right)^{1 / 2}\right]^{2} \\
& \leq\left(\left\|\lambda_{n} x_{0}\right\|+\left\|z_{0}\right\|\right)^{2} \\
&=\left(\left|\lambda_{n}\right|+\left\|z_{0}\right\|\right)^{2} \cdot \\
& \sum_{i \in A_{3}}\left|\mathcal{I}_{i}^{*}\left(z_{n}-z_{0}\right)\right|^{2}=\sum_{i \in A_{3}}\left|\mathcal{I}_{i}^{*}\left(x_{n}\right)+\mathcal{I}_{i}^{*}\left(-\lambda_{n} x_{0}\right)-\mathcal{I}_{i}^{*}\left(z_{0}\right)\right|^{2} \\
& \leq\left[\left(\sum_{i \in A_{3}}\left|\mathcal{I}_{i}^{*}\left(x_{n}\right)\right|^{2}\right)^{1 / 2}+\left\|\lambda_{n} x_{0}\right\|+\left\|z_{0}\right\|\right]^{2} \\
& \leq\left[\left(2^{m} \frac{1}{2^{n}}\right)^{1 / 2}+\left|\lambda_{n}\right|+\left\|z_{0}\right\|\right]^{2} \\
& \leq\left[\left(1-\frac{1}{2^{m}}+\frac{1}{2^{n}}\right)^{1 / 2}+\left|\lambda_{n}\right|\right]^{2} \\
& \sum_{i \in A_{2}}\left|\mathcal{I}_{i}^{*}\left(z_{n}-z_{0}\right)\right|^{2}=\sum_{i \in A_{2}}\left|\mathcal{I}_{i}^{*}\left(z_{n}\right)\right|^{2} \\
&=\sum_{i \in A_{2}}\left|\mathcal{I}_{i}^{*}\left(x_{n}\right)+\mathcal{I}_{i}^{*}\left(-\lambda_{n} x_{0}\right)\right|^{2} \\
& \leq\left[\left(\sum_{i \in A_{2}}\left|\mathcal{I}_{i}^{*}\left(x_{n}\right)\right|^{2}\right)^{1 / 2}+\left|\lambda_{n}\right|\right]^{2} \\
&\left.\leq\left[\left(2^{n}-2^{n-k}+1\right) \frac{1}{2^{n}}\right)^{1 / 2}+\left|\lambda_{n}\right|\right]^{2} \\
&=[(1 / 2 \\
& 2^{k} \\
&
\end{aligned}
$$

Therefore, 


$$
\begin{aligned}
\sum_{i=1}^{r}\left|\mathcal{I}_{i}^{*}\left(z_{n}-z_{0}\right)\right|^{2} \leq\left(\left|\lambda_{n}\right|+\left\|z_{0}\right\|\right)^{2} & +\left[\left(\frac{2^{m}}{2^{n}}\right)^{1 / 2}+\left|\lambda_{n}\right|+\left\|z_{0}\right\|\right]^{2} \\
+ & {\left[\left(1-\frac{1}{2^{m}}+\frac{1}{2^{n}}\right)^{1 / 2}+\left|\lambda_{n}\right|\right]^{2} . }
\end{aligned}
$$

Finally, we have

$$
\begin{aligned}
\left\|z_{n}-z_{0}\right\|^{2} \leq \max \left\{\left\|z_{n}\right\|^{2},\left(\left|\lambda_{n}\right|\right.\right. & \left.+\left\|z_{0}\right\|\right)^{2}+\left[\left(\frac{2^{m}}{2^{n}}\right)^{1 / 2}+\left|\lambda_{n}\right|+\left\|z_{0}\right\|\right]^{2} \\
& \left.+\left[\left(1-\frac{1}{2^{m}}+\frac{1}{2^{n}}\right)^{1 / 2}+\left|\lambda_{n}\right|\right]^{2}\right\} .
\end{aligned}
$$

As $n$ tends to infinity, the sum

$$
\left(\left|\lambda_{n}\right|+\left\|z_{0}\right\|\right)^{2}+\left[\left(\frac{2^{m}}{2^{n}}\right)^{1 / 2}+\left|\lambda_{n}\right|+\left\|z_{0}\right\|\right]^{2}+\left[\left(1-\frac{1}{2^{m}}+\frac{1}{2^{n}}\right)^{1 / 2}+\left|\lambda_{n}\right|\right]^{2}
$$

converges to $2\left\|z_{0}\right\|^{2}+1-\left(1 / 2^{m}\right)$. By the choice of $z_{0}$ we have $2\left\|z_{0}\right\|^{2}+1-\left(1 / 2^{m}\right)<1$. Therefore, $\lim _{n \rightarrow \infty}\left\|z_{n}-z_{0}\right\|=1=$ $\lim _{n \rightarrow \infty}\left\|z_{n}\right\|$, and the proof is complete.

By Proposition 3.4 (respectively, 6.3) we know that $X$ (respectively, $Y$ ) contains subspaces which are isometrically isomorphic to $\ell_{2}$. Therefore, there are infinite dimensional subspaces of $X$ (respectively, $Y$ ) satisfying the Opial condition. However, we expect that Theorem 7.2 can be expanded to a wider class of subspaces of $X$ (respectively, $Y$ ). So, we state the next problem.

Problem 7.3. Does any subspace of $X$ (respectively, $Y$ ) with nonseparable dual fail the Opial property?

Remark 7.4. As it was mentioned in the introduction, the class of separable Banach spaces not containing $\ell_{1}$ with non-separable dual was established by two well-known examples: the James tree space $(J T)$ and the James function space $(J F)$. The first one satisfies the Opial condition. We now prove that the space $J F$ does not satisfy this condition. In order to do this, we use the fact that $J F$ is isometric to the space $V_{2}^{0}$ (see $\left.[\mathbf{2}, \mathbf{1 1}]\right)$. We also use the notation from [2]. 
Theorem 7.5. The space JF does not satisfy the Opial condition.

Proof. We set $R_{n}(t)=2^{n / 2} \int_{0}^{t} r_{n}(x) d x$, where $\left\{r_{n}\right\}_{n=0}^{\infty}$ denotes the sequence of Rademacher functions. Then, we have $\left\|R_{n}\right\|=1$ and $\left\|R_{n}\right\|_{\infty} \rightarrow 0$. Since the weak and the pointwise topology coincide on bounded subsets of $V_{2}^{0}$, it follows that $\left\{R_{n}\right\}_{n \in \mathbb{N}}$ is weakly null. We now consider the identity map $f(x)=x$ on $[0,1]$, and we show that $\liminf \left\|f+R_{n}\right\| \leq 1$.

Given $\epsilon>0$, we fix $\delta>0$ such that, for any family $\mathcal{I}$ of pairwise disjoint open intervals of $[0,1]$ with $|I|<\delta$ for every $I \in \mathcal{I}, v_{2}(f, \mathcal{I})<\epsilon$. For every $n \in \mathbb{N}$, we choose a family $\mathcal{I}_{n}$ of pairwise disjoint open intervals of $[0,1]$ such that

$$
\begin{aligned}
\left\|f+R_{n}\right\|^{2} & =v_{2}^{2}\left(f+R_{n}, \mathcal{I}_{n}\right) \\
& =v_{2}^{2}\left(f+R_{n}, \mathcal{I}_{n}^{\geq \delta}\right)+v_{2}^{2}\left(f+R_{n}, \mathcal{I}_{n}^{<\delta}\right),
\end{aligned}
$$

where $\mathcal{I}_{n}^{\geq \delta}=\left\{I \in \mathcal{I}_{n}:|I| \geq \delta\right\}$ and $\mathcal{I}_{n}^{<\delta}=\left\{I \in \mathcal{I}_{n}:|I|<\delta\right\}$. Since $\left\|R_{n}\right\|_{\infty} \rightarrow 0$, there is $n_{0} \in \mathbb{N}$, such that, for every $n>n_{0}$, $v_{2}\left(R_{n}, \mathcal{I}_{\bar{n}}^{\geq \delta}\right)<\epsilon$. We also make use of the following inequalities $v_{2}^{2}\left(R_{n}, \mathcal{I}_{n}^{<\delta}\right) \leq \lambda\left(\cup \mathcal{I}_{n}^{<\delta}\right), v_{2}^{2}\left(f, \mathcal{I}_{\bar{n}}^{\geq \delta}\right) \leq \lambda\left(\cup \mathcal{I}_{n}^{\geq \delta}\right), v_{2}\left(f+R_{n}, \mathcal{I}_{n}^{\geq \delta}\right) \leq$ $v_{2}\left(f, \mathcal{I}_{n}^{\geq \delta}\right)+v_{2}\left(R_{n}, \mathcal{I}_{\bar{n}}^{\geq \delta}\right), v_{2}\left(f+R_{n}, \mathcal{I}_{n}^{<\delta}\right) \leq v_{2}\left(f, \mathcal{I}_{n}^{<\delta}\right)+v_{2}\left(R_{n}, \mathcal{I}_{n}^{<\delta}\right)$, where $\lambda(A)$ denotes the length (that is, Lebesgue measure) of the set $A \subseteq[0,1]$. Finally, we get

$$
\left\|f+R_{n}\right\|^{2} \leq \lambda\left(\cup \mathcal{I}_{n}^{\geq \delta}\right)+\lambda\left(\cup \mathcal{I}_{n}^{<\delta}\right)+2 \epsilon^{2}+4 \epsilon \leq 1+6 \epsilon,
$$

for every $n>n_{0}$. Therefore, $\liminf \left\|f+R_{n}\right\| \leq 1$.

In view of Theorem 7.5 , it is natural to ask the following.

Problem 7.6. Does the space JF satisfy the fixed point property?

Remark 7.7. An important member of the class of separable Banach spaces not containing $\ell_{1}$ with non-separable dual is the so-called Hagler tree space $(H T)$ (see [6]). This space is $c_{0}$-saturated, that is, any closed, infinite dimensional subspace of $H T$ contains an isomorphic copy of $c_{0}$. It is easily verified that $H T$ does not satisfy the Opial condition. However, we do not know if $H T$ has the fixed point property. 
Acknowledgments. The author would like to thank the referee for his valuable comments and suggestions. In particular, the proof of Theorem 7.5 is due to the referee.

\section{REFERENCES}

1. D. Alspach, A fixed point free nonexpansive map, Proc. Amer. Math. Soc. 82 (1981), 423-424.

2. D. Apatsidis, S.A. Argyros and V. Kanellopoulos, On the subspaces of JF and JT with non-separable dual, J. Funct. Anal. 254 (2008), 632-674.

3. F.E. Browder, Nonexpansive nonlinear operators in Banach spaces, Proc. Nat. Acad. Sci. 54 (1965), 1041-1044.

4. J. Elton, P. Lin, E. Odell and S. Szarek, Remarks on the fixed point problem for nonexpansive maps, Contemp. Math. 18 (1983), 87-119.

5. J.P. Gossez and E. Lami Dozo, Some geometric properties related to the fixed point theory for nonexpansive mappings, Pac. J. Math. 40 (1972), 565-573.

6. J. Hagler, A counterexample to several questions about Banach spaces, Stud. Math. 60 (1977), 289-308.

7. R.C. James, A separable somewhat reflexive Banach space with non-separable dual, Bull. Amer. Math. Soc. 80 (1974), 738-743.

8. L.A. Karlovitz, Existence of fixed points for nonexpansive mappings in a space without normal structure, Pac. J. Math. 66 (1976), 153-159.

9. M.A. Khamsi, James' quasi-reflexive space has the fixed point property, Bull. Austr. Math. Soc. 39 (1989), 25-30.

10. W.A. Kirk, A fixed point theorem for mappings which do not increase distances, Amer. Math. Month. 72 (1965), 1004-1006.

11. S.V. Kisliakov, A remark on the space of functions of bounded p-variation, Math. Nachr. 119 (1984), 37-40.

12. T. Kuczumow and S. Reich, Opial's property and James' quasi-reflexive spaces, Comm. Math. Univ. Carolin. 35 (1994), 283-289.

13. J. Lindenstrauss and C. Stegall, Examples of separable spaces which do not contain $\ell_{1}$ and whose duals are nonseparable, Stud. Math. 54 (1975), 81-105.

14. B. Maurey, Points fixes des contractions sur un convexe forme de $L^{1}$, Semi. Anal. Fonction. 80-81, Ecole Polytechnique, Palaiseau.

15. Z. Opial, Weak convergence of the sequence of successive approximations for nonexpansive mappings, Bull. Amer. Math. Soc. 73 (1967), 591-597.

Department of Mathematics, University of Athens, 15784, Athens, Greece Email address: costas314@gmail.com 